\title{
Bipolar Imidazolium-based Lipid Analogues for Artificial Archaeosomes
}

\author{
Simon Kriegler ${ }^{\text {a† }}$, Tiffany O. Paulisch ${ }^{\text {b }}$, Tristan Wegner ${ }^{\mathrm{b}}$, Frank Glorius ${ }^{\mathrm{b}^{*}}$, Roland \\ Winter ${ }^{a^{*}}$ \\ a TU Dortmund University, Department of Chemistry and Chemical Biology, Physical \\ Chemistry I - Biophysical Chemistry, Otto Hahn Str. 4a, D-44221 Dortmund, \\ Germany; *roland.winter@tu-dortmund.de \\ b University of Münster, Institute of Organic Chemistry, Corrensstraße 40, 48149 \\ Münster, Germany; *glorius@uni-muenster.de \\ † These authors contributed equally.
}

\section{Supplementary Information}

\section{Organic synthesis}

\subsection{General considerations}

Reactions were performed in flame-dried glassware under an atmosphere of dry argon, unless otherwise noted. The given reaction temperatures correspond to the temperature of the preheated oil bath surrounding the reaction vessel. The solvents were either purified via distillation over standard drying agents under an atmosphere of dry argon or purchased dry from Acros Organics, Carl Roth or Sigma-Aldrich and stored over molecular sieves. Solvents used for extraction, crystallization or column chromatography were technical grade and flashdistilled prior to use. All commercial chemicals and reagents were purchased from $A B C R$, Acros Organics, Alfa Aesar, Combi-Blocks, Fluorochem, Merck, Sigma Aldrich, TCI Europe or $V W R$ and were used without further purification. Brine denotes as sat. aq. solution of sodium chloride.

Monitoring of the reaction progress was performed by thin layer chromatography on precoated aluminum-backed plates (Merck Kieselgel 60 F254). Detection of the compounds was performed by irradiation with light $(254 \mathrm{~nm}$ or $366 \mathrm{~nm})$ or using potassium permanganate as a staining solution. Purification via flash column chromatography was performed with Acros silica gel $(35-70 \mu \mathrm{m}, 60 \AA)$ with a head pressure of argon $(\sim 0.2$ atmospheres). The ratio of the solvent mixture is given with the reaction procedure.

NMR-spectra were recorded on a Bruker ARX-300, AV-300 or AV-400 MHz. The signals are expressed as chemical shifts $(\delta)$ in parts per million $(\mathrm{ppm})$ relative to tetramethylsilane with the 
corresponding solvent as the reference for ${ }^{1} \mathrm{H}$ and ${ }^{13} \mathrm{C}$ NMR spectra. As references for ${ }^{1} \mathrm{H}$ and ${ }^{13} \mathrm{C}$ NMR-spectra $\mathrm{CDCl}_{3}\left(\delta_{\mathrm{H}}=7.26 \mathrm{ppm} ; \delta_{\mathrm{C}}=77.16 \mathrm{ppm}\right)$ was used. Coupling constants $(\mathrm{J})$ are quoted in $\mathrm{Hz}$. The following abbreviations are used for multiplicities of signals: $\mathrm{s}$ (singlet), $d$ (doublet), t (triplet), q (quartet), p (pentet), m (multiplet) or combinations of these.

ESI-MS spectra were obtained using a Bruker Daltonics MicroTof or Thermo Scientific Orbitrap LTQ XL. The instrument was calibrated using sodium formicates clusters. GC-MS chromatograms were recorded on an Agilent Technologies 7890A GC system with an HP5MS column. A standard separation method $\left(50^{\circ} \mathrm{C}\right.$ for $3 \mathrm{~min}$, heated to $290^{\circ} \mathrm{C}$ by $40{ }^{\circ} \mathrm{C} / \mathrm{min}$, $290{ }^{\circ} \mathrm{C}$ for $3 \mathrm{~min}$ ) was used. The GC system is coupled with an Agilent 5975C VL mass selective detector (MSD) or an Agilent 5975 inert mass selective detector for recording El mass spectra. The major signals of are quoted as $\mathrm{m} / \mathrm{z}$. UPLC-UV/MS measurements were recorded on a Waters Acquity UPLC H-Class Series system with an Acquity UPLC BEH C18 $1.7 \mu \mathrm{m} 2.1$ x $50 \mathrm{~mm}$ column and $15 \mu \mathrm{L}$ injection needle. As standard method, a solvent gradient variation of $\mathrm{H}_{2} \mathrm{O}$ :MeCN with $0.1 \mathrm{v} \%$ formic acid was applied with column temperature $40{ }^{\circ} \mathrm{C}$, flow 0.6 $\mathrm{mL} / \mathrm{min}$, injection volume $10 \mu \mathrm{L}$, duration $5 \mathrm{~min}$. UV-VIS absorption was detected at $254 \mathrm{~nm}$ using a Waters Acquity UPLC tunable UV detector. ESI-MS ion counts were detected between $80 \mathrm{~m} / \mathrm{z}$ and $400 \mathrm{~m} / \mathrm{z}$ with a cone voltage of $25 \mathrm{~V}$ in positive ionization mode at $10 \mathrm{~Hz}$ preset using a Waters Acquity UPLC QDa detector.

\subsection{Experimental}

\subsubsection{BImN10 / BImC10}<smiles>Cn1ccnc1</smiles><smiles>BrCCCCCCCCCCCBr</smiles>

$$
\downarrow \begin{gathered}
\mathrm{THF} \\
\downarrow 100^{\circ} \mathrm{C}, \text { on }
\end{gathered}
$$

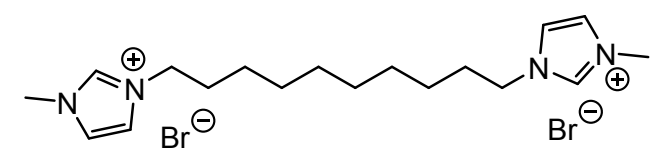

BImN10

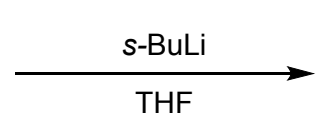

$-78{ }^{\circ} \mathrm{C} \rightarrow \mathrm{rt}, 1+2.5 \mathrm{~h}$

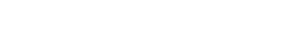

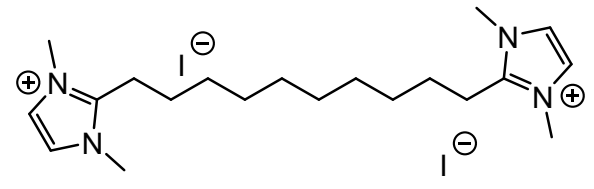

BImC10 
1.2.1.1 1-Methyl-3-(10-(3-methylimidazolium-1-yl)decyl)-imidazolium bromide

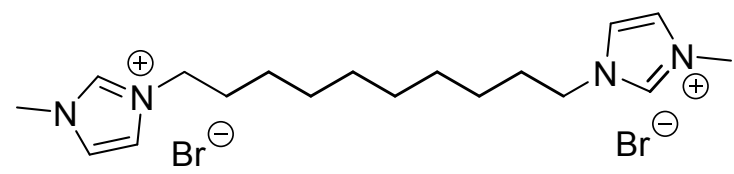

Decan-1,10-dibromide ( $0.11 \mathrm{~mL}, 0.50 \mathrm{mmol}, 1.0$ equiv) and 1-methyl imidazole (88 $\mu \mathrm{L}$, $1.10 \mathrm{mmol}, 2.2$ equiv) were dissolved in dry $\operatorname{THF}(2 \mathrm{~mL})$ and stirred at $100^{\circ} \mathrm{C}$ overnight. The solvent was removed in vacuo and residue washed with diethylether. The product (199 $\mathrm{mg}, 0.4 \mathrm{mmol}, 86 \%$ ) was isolated as white resin.

${ }^{1} \mathrm{H}$ NMR (300 MHz, MeOD) $\delta(\mathrm{ppm}): 9.01$ (s, N-CH-N, 2H), $7.70-7.63$ (m, N-CH-CH, 2H), 7.62 - 7.55 (m, N-CH-CH, 2H), 4.22 (t, J = 7.3 Hz, N-CH2-CH2, 4H), 3.94 (s, CH3$\mathrm{N}, 6 \mathrm{H}), 1.97$ - 1.81 (m, NCH2-CH2-CH2, 4H), $1.40-1.29(\mathrm{~m}, \mathrm{CH} 2-\mathrm{CH} 2-\mathrm{CH} 2,12 \mathrm{H})$. ${ }^{13} \mathrm{C}$ NMR (75 MHz, $\left.\mathrm{CDCl}_{3}\right) \delta$ (ppm): $137.9(\mathrm{~N}-\mathrm{CH}-\mathrm{N}), 124.9(\mathrm{~N}-\mathrm{CH}-\mathrm{CH}), 123.7(\mathrm{~N}-\mathrm{CH}-$ $\mathrm{CH}), 50.8$ (N-CH2-CH2), $36.5(\mathrm{CH} 3-\mathrm{N}), 31.2(\mathrm{CH} 2-\mathrm{CH} 2-\mathrm{CH} 2), 30.4(\mathrm{CH} 2-\mathrm{CH} 2-\mathrm{CH} 2)$, $30.0(\mathrm{CH} 2-\mathrm{CH} 2-\mathrm{CH} 2), 27.2(\mathrm{CH} 2-\mathrm{CH} 2-\mathrm{CH} 2)$.

HRMS (ESI): $m / z$ calculated for $[M]^{2+}\left(\mathrm{C}_{18} \mathrm{H}_{32}{ }^{2+}\right): 152.1308$, found: 152.1296 .

\subsubsection{1,10-bis(1-methylimidazol-2-yl)decane}

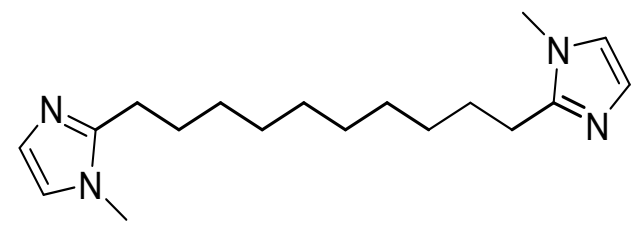

1-Methylimidazole $(351 \mu \mathrm{L}, 4.40 \mathrm{mmol}, 2.2$ equiv) was suspended in dry THF (14.6 mL) and cooled to $-78{ }^{\circ} \mathrm{C}$. $s$-BuLi (1.6 M, $2.75 \mathrm{~mL}, 4.40 \mathrm{mmol}, 2.2$ equiv) was added dropwise and the reaction mixture was stirred for $1 \mathrm{~h}$ at $-78^{\circ} \mathrm{C}$. Following, decan-1,10-dibromide (450 $\mu \mathrm{L}, 2.00 \mathrm{mmol}, 1.0$ equiv) was added dropwise and the reaction was allowed to warm to rt. After stirring the reaction mixture for $2.5 \mathrm{~h}$ water was added and the mixture extracted with diethylether. The combined organic layers were dried over $\mathrm{MgSO}_{4}$, filtered, and concentrated in vacuo. Purification by column chromatography $\left(\mathrm{SiO}_{2}\right.$; DCM:MeOH 9:1) yielded the product $(109 \mathrm{mg}, 0.36 \mathrm{mmol}$, $18 \%)$ as colorless oil.

$\mathbf{R}_{\mathbf{f}}$ (DCM:MeOH, 90:10): 0.85. 
${ }^{1} \mathrm{H}$ NMR (400 MHz, MeOD) $\delta(\mathrm{ppm}): 6.93$ (d, J = $\left.1.4 \mathrm{~Hz}, \mathrm{~N}-\mathrm{CH}-\mathrm{CH}, 2 \mathrm{H}\right), 6.80$ (d, J = $1.4 \mathrm{~Hz}, \mathrm{~N}-\mathrm{CH}-\mathrm{CH}, 2 \mathrm{H}$ ), 3.61 (s, CH3-N, 6H), $2.72-2.64$ (m, C-CH2-CH2, 4H), $1.73-$ 1.61 (m, CCH2-CH2-CH2, 4H), 1.39 - 1.23 (m, CH2- $\mathrm{CH} 2-\mathrm{CH} 2,12 \mathrm{H})$.

${ }^{13} \mathrm{C}$ NMR (101 MHz, MeOD) $\delta$ (ppm): $150.0(\mathrm{~N}-\mathrm{C}-\mathrm{N}), 126.6(\mathrm{~N}-\mathrm{CH}-\mathrm{CH}), 121.9(\mathrm{~N}-\mathrm{CH}-$ $\mathrm{CH}), 33.0(\mathrm{CH} 3-\mathrm{N}), 30.6(\mathrm{CH} 2-\mathrm{CH} 2-\mathrm{CH} 2), 30.4(\mathrm{CH} 2-\mathrm{CH} 2-\mathrm{CH} 2), 30.3(\mathrm{CH} 2-\mathrm{CH} 2-\mathrm{CH} 2)$, 29.0( $(\mathrm{CH} 2-\mathrm{CH} 2-\mathrm{C}), 27.2(\mathrm{CH} 2-\mathrm{CH} 2-\mathrm{CH} 2)$.

HRMS (ESI): $m / z$ calculated for $[\mathrm{M}+\mathrm{H}]^{+}\left(\mathrm{C}_{18} \mathrm{H}_{31} \mathrm{~N}_{4}{ }^{+}\right): 303.2543$, found: 303.2553 .

1.2.1.3 2,2'-(decane-1,10-diyl)bis(1,3-dimethylimidazolium) iodide

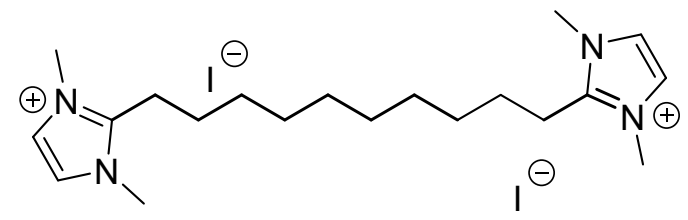

1,10-Bis(1-methylimidazol-2-yl)decane $\quad(109 \mathrm{mg}, \quad 0.40 \mathrm{mmol}, \quad 1.0$ equiv) was suspended in dry acetonitrile $(0.72 \mathrm{~mL})$. Methyliodide (56 $\mu \mathrm{L}, 0.90 \mathrm{mmol}, 2.5$ equiv) was added and the reaction mixture heated at $85^{\circ} \mathrm{C}$ overnight. The mixture was filtered and the solid washed with diethylether. The product (142 mg, $0.24 \mathrm{mmol}, 67 \%$ ) was isolated as colourless solid.

${ }^{1} \mathrm{H}$ NMR (400 MHz, $\mathrm{CDCl}_{3}$ ) $\delta(\mathrm{ppm}): 7.48-7.43(\mathrm{~m}, \mathrm{~N}-\mathrm{CH}-\mathrm{CH}, 4 \mathrm{H}), 3.87-3.83(\mathrm{~m}$, CH3-N, 12H), $3.09-3.00$ (m, C-CH2-CH2, 4H), $1.74-1.63(\mathrm{~m}, \mathrm{CCH} 2-\mathrm{CH} 2-\mathrm{CH} 2,4 \mathrm{H})$, $1.50-1.31(\mathrm{~m}, \mathrm{CH} 2-\mathrm{CH} 2-\mathrm{CH} 2,12 \mathrm{H})$.

${ }^{13} \mathrm{C}$ NMR (101 MHz, $\left.\mathrm{CDCl}_{3}\right) \delta(\mathrm{ppm}): 149.0(\mathrm{~N}-\mathrm{C}-\mathrm{N}), 123.6(\mathrm{~N}-\mathrm{CH}-\mathrm{CH}), 35.7(\mathrm{CH}-\mathrm{N})$, 30.5( $\mathrm{CH} 2-\mathrm{CH} 2-\mathrm{CH} 2), 30.3(\mathrm{CH} 2-\mathrm{CH} 2-\mathrm{CH} 2), 30.2\left(\mathrm{CH} 2-\mathrm{CH}_{2}-\mathrm{CH} 2\right), 27.4\left(\mathrm{CH}_{2}-\mathrm{CH} 2-\mathrm{C}\right)$, 24.1(CH2-CH2-CH2).

HRMS (ESI): $m / z$ calculated for $[M]^{2+}\left(\mathrm{C}_{20} \mathrm{H}_{36} \mathrm{~N}_{4}{ }^{2+}\right): 166.1465$, found: 166.1479 . 


\subsubsection{BImN16}
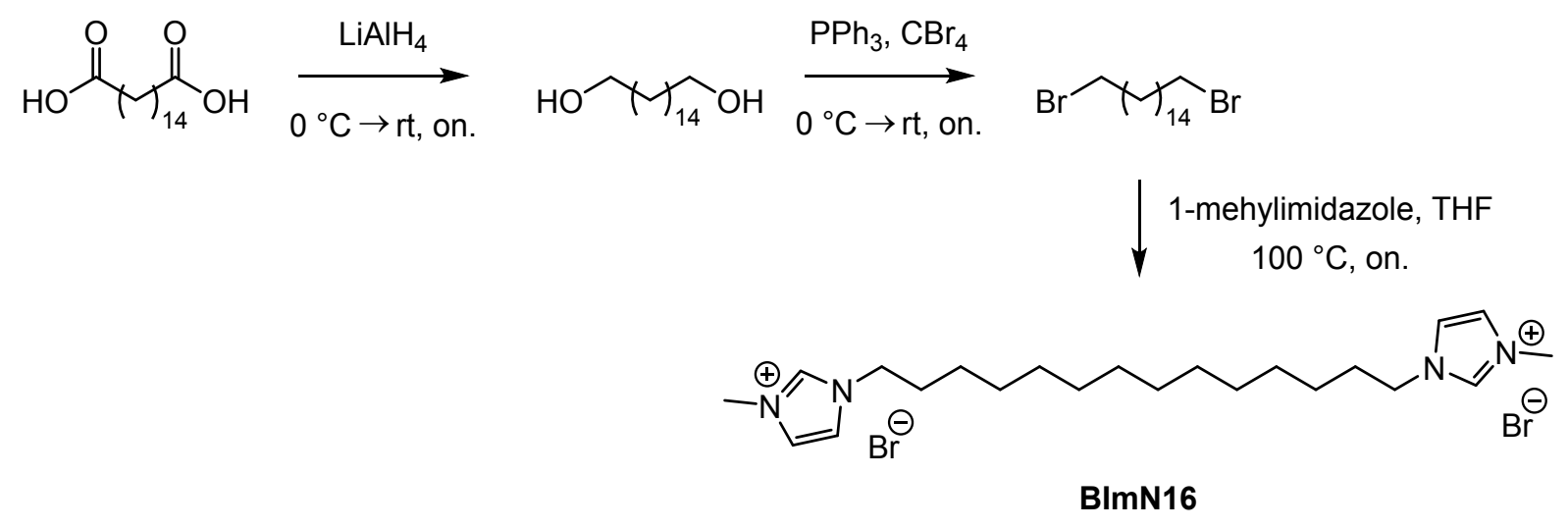

1.2.2.1 Hexadecane-1,16-diol ${ }^{[1,2]}$

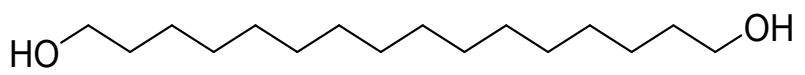

Lithium aluminium hydride ( $759 \mathrm{mg}, 20.0 \mathrm{mmol}, 4.0$ equiv) was suspended in THF $(20 \mathrm{~mL})$ and cooled to $0{ }^{\circ} \mathrm{C}$. Following, hexadecanedioic acid $(1.43 \mathrm{~g}, 5.00 \mathrm{mmol}$, 1.0 equiv) was added portionwise and stirred at rt overnight. The reaction mixture was quenched with water at $0{ }^{\circ} \mathrm{C}$ and extracted with diethylether. The combined organic layers were dried over $\mathrm{MgSO}_{4}$, filtered and concentrated in vacuo. Purification by column chromatography $\left(\mathrm{SiO}_{2}\right.$; pentane/EtOAc 19/1) yielded the product (956 mg, $3.70 \mathrm{mmol}, 74 \%$ ) as white solid.

$\mathbf{R}_{\mathbf{f}}$ (pentane:EtOAc, 19:1): 0.61.

${ }^{1} \mathrm{H}$ NMR (400 MHz, MeOD) $\delta(\mathrm{ppm}): 3.56$ (t, J = 6.6 Hz, CH2-OH), $1.59-1.49$ (m, CH2$\mathrm{CH} 2-\mathrm{OH}, 4 \mathrm{H}), 1.43-1.30$ (m, CH2-CH2-CH2, 26H).

${ }^{13} \mathrm{C}$ NMR (101 MHz, $\left.\mathrm{CDCl}_{3}\right) \delta(\mathrm{ppm}): 63.0(\mathrm{CH} 2-\mathrm{CH} 2-\mathrm{OH}), 33.7(\mathrm{CH} 2-\mathrm{CH} 2-\mathrm{CH} 2), 30.8$ (CH2-CH2-CH2), 30.7 (CH2- $\mathrm{CH} 2-\mathrm{CH} 2), 30.6$ (CH2-CH2-CH2), $27.0(\mathrm{CH} 2-\mathrm{CH} 2-\mathrm{CH} 2)$. 2 signals missing due to overlap

HRMS (ESI): $\mathrm{m} / \mathrm{z}$ calculated for $[\mathrm{M}+\mathrm{Na}]^{+}\left(\mathrm{C}_{16} \mathrm{H}_{34} \mathrm{O}_{2} \mathrm{Na}^{+}\right): 281.2451$, found: 281.2449 .

\subsubsection{1,16-Dibromohexadecane ${ }^{[3,4]}$}

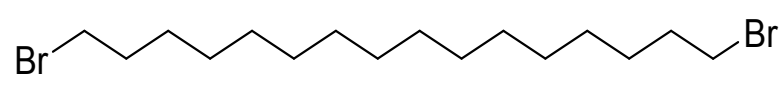

Hexadecane-1,16-diol (258 mg, $1.00 \mathrm{mmol}, 1.0$ equiv.) and triphenyl phosphine (944 mg, $3.60 \mathrm{mmol}, 3.6$ equiv) were dissolved in THF $\left(5.4 \mathrm{~mL}\right.$ ) and cooled to $0{ }^{\circ} \mathrm{C}$. 
After addition of tetrabromo methane $(1.06 \mathrm{~g}, 3.20 \mathrm{mmol}, 3.2$ equiv) the reaction mixture was stirred at rt overnight. The reaction was filtered, rinsed with diethylether and concentrated in vacuo. Purification by by column chromatography $\left(\mathrm{SiO}_{2} ;\right.$ pentane) yielded the product (293 $\mathrm{mg}, 0.76 \mathrm{mmol}, 76 \%$ ) as white solid.

$\mathbf{R}_{\mathbf{f}}$ (pentane): 0.90 .

${ }^{1} \mathrm{H}$ NMR (400 MHz, MeOD) $\delta(\mathrm{ppm}): 3.46(\mathrm{t}, \mathrm{J}=6.8 \mathrm{~Hz}, \mathrm{CH} 2-\mathrm{CH} 2-\mathrm{Br}, 4 \mathrm{H}), 1.92-1.80$ (m, BrCH2- $\mathrm{CH} 2-\mathrm{CH} 2,4 \mathrm{H}), 1.51-1.41(\mathrm{~m}, \mathrm{BrC2H} 4-\mathrm{CH} 2-\mathrm{CH} 2,4 \mathrm{H}), 1.39-1.30$ (m, $\mathrm{CH} 2-\mathrm{CH} 2-\mathrm{CH} 2,2 \mathrm{OH})$.

${ }^{13} \mathrm{C}$ NMR (101 MHz, MeOD) $\delta$ (ppm): 34.4 (CH2-CH2-CH2), $34.0(\mathrm{CH} 2-\mathrm{CH} 2-\mathrm{CH} 2)$, 30.72 (CH2-CH2-CH2), 30.69 (CH2-CH2-CH2), 30.63 (CH2-CH2-CH2), 30.57 (CH2$\mathrm{CH} 2-\mathrm{CH} 2), 29.8$ (CH2-CH2-CH2), $29.2(\mathrm{CH} 2-\mathrm{CH} 2-\mathrm{CH} 2)$.

GC-MS: $m / z$ calculated for [M] $\left(\mathrm{C}_{16} \mathrm{H}_{32} \mathrm{Br}_{2}\right)$ : 384.09 , found: 384.0 .

1.2.2.3 1,1'-(Hexadecane-1,16-diyl)bis(3-methylimidazolium) bromide

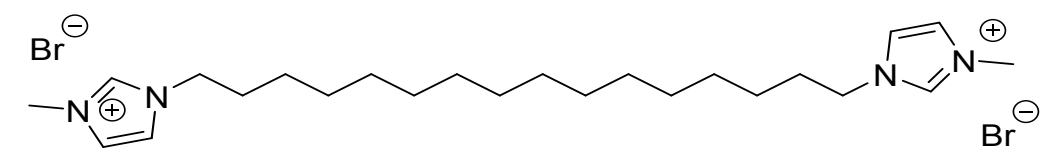

1,16-Dibromohexadecane (293 mg, $0.76 \mathrm{mmol}, 1.0$ equiv) and 1-methyl imidazole (138 mg, $1.67 \mathrm{mmol}, 2.2$ equiv) were dissolved in THF $(3.3 \mathrm{~mL}$ ) and heated overnight at $100{ }^{\circ} \mathrm{C}$. Following, the mixture was filtered and the residue was washed with diethyl ether. The product $(41.7 \mathrm{mg}, 0.08 \mathrm{mmol}, 10 \%)$ was isolated as white resin.

${ }^{1} \mathrm{H}$ NMR (300 MHz, MeOD) $\delta(\mathrm{ppm}): 8.99$ (s, N-CH-N, 2H), $7.70-7.66(\mathrm{~m}, \mathrm{~N}-\mathrm{CH}-\mathrm{CH}$, 2H), $7.62-7.58$ (m, N-CH-CH, 2H), 4.24 (t, J = 7.4 Hz, N-CH2-CH2, 4H), 3.96 (s, CH3$\mathrm{N}, 6 \mathrm{H}), 1.99-1.86(\mathrm{~m}, \mathrm{NCH} 2-\mathrm{CH} 2-\mathrm{CH} 2,4 \mathrm{H}), 1.44-1.26(\mathrm{~m}, \mathrm{CH} 2-\mathrm{CH} 2-\mathrm{CH} 2,24 \mathrm{H})$.

${ }^{13} \mathrm{C}$ NMR (75 MHz, MeOD) $\delta(p p m): 137.9(\mathrm{~N}-\mathrm{CH}-\mathrm{N}), 125.0(\mathrm{~N}-\mathrm{CH}-\mathrm{CH}), 123.7(\mathrm{~N}-\mathrm{CH}-$ $\mathrm{CH}), 50.9(\mathrm{~N}-\mathrm{CH} 2-\mathrm{CH} 2), 36.5(\mathrm{CH} 3-\mathrm{N}), 31.2(\mathrm{CH} 2-\mathrm{CH} 2-\mathrm{CH} 2), 30.84(\mathrm{CH} 2-\mathrm{CH} 2-\mathrm{CH} 2)$, 30.80 (CH2-CH2-CH2), 30.7 (CH2- $\mathrm{CH} 2-\mathrm{CH} 2), 30.6$ (CH2-CH2-CH2), $30.2(\mathrm{CH} 2-\mathrm{CH} 2-$ $\mathrm{CH} 2), 27.3(\mathrm{CH} 2-\mathrm{CH} 2-\mathrm{CH} 2)$.

HRMS (ESI): $\mathrm{m} / \mathrm{z}$ calculated for [M] ${ }^{+}\left(\mathrm{C}_{24} \mathrm{H}_{44} \mathrm{~N}_{4} \mathrm{Br}^{+}\right): 467.2749$, found: 467.2744 . 


\subsubsection{BImN32}

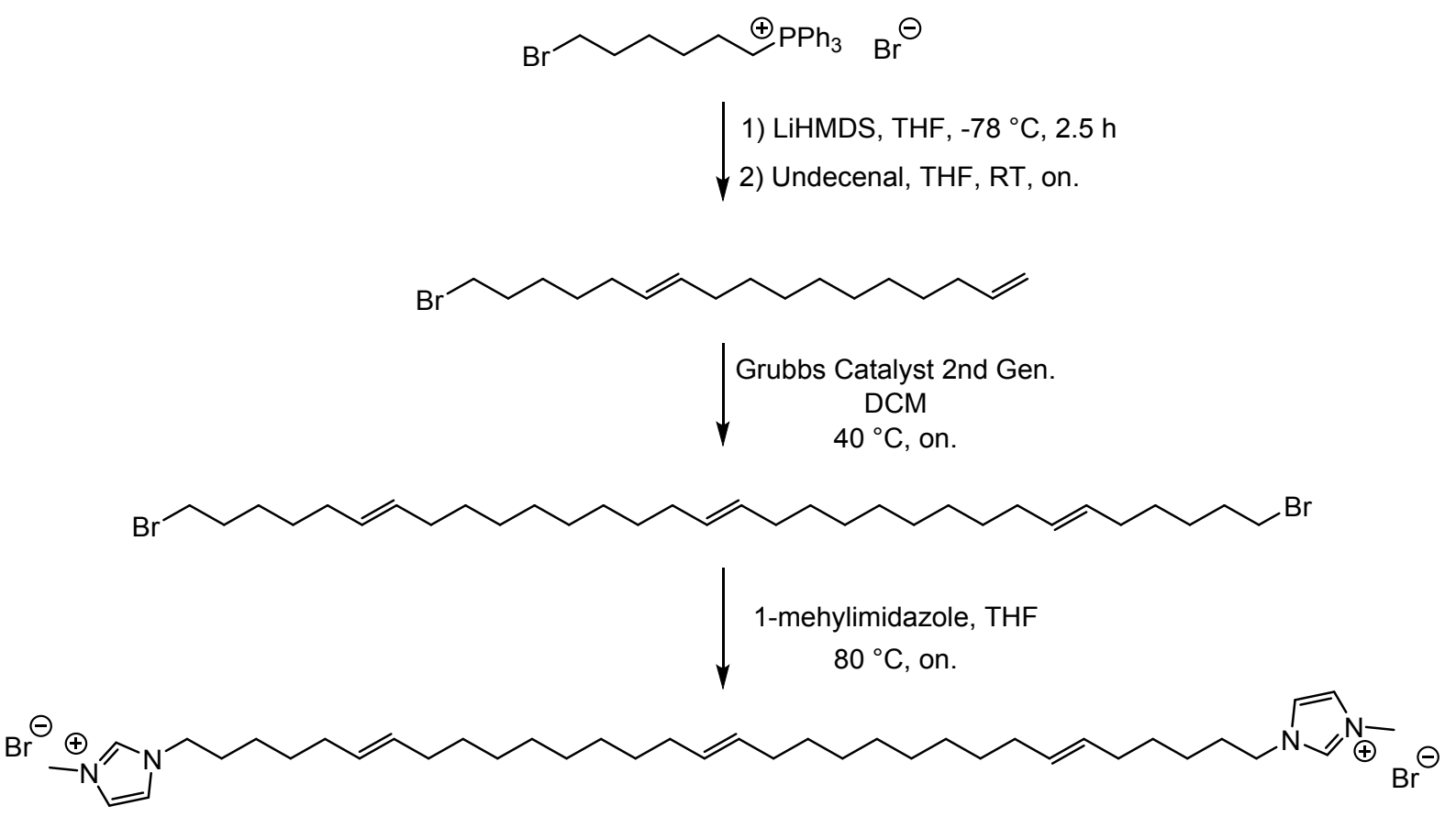

1.2.3.1 17-Bromoheptadeca-1,11-diene

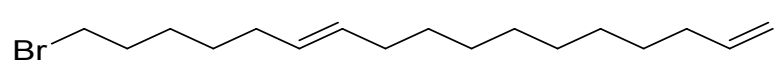

(6-Bromohexyl) triphenyl-phosphonium bromide (717 mg, $1.40 \mathrm{mmol}, 1.2$ equiv) was suspended in dry THF and cooled to $-78{ }^{\circ} \mathrm{C}$. LiHMDS $(1 \mathrm{M}, 1.3 \mathrm{~mL}, 1.30 \mathrm{mmol}$, 1.1 equiv) was added dropwise and reaction mixture was stirred $2.5 \mathrm{~h}$ at $-78{ }^{\circ} \mathrm{C}$. Following, undec-10-enal (236.5 $\mu \mathrm{L}, 1.20 \mathrm{mmol}, 1.0$ equiv) was added and stirred at $\mathrm{rt}$ overnight. Water was added and the reaction mixture was extracted with EtOAc. The combined organic layers were dried over $\mathrm{MgSO}_{4}$, filtered and concentrated in vacuo. Purification by column chromatography $\left(\mathrm{SiO}_{2}\right.$; pentane) yielded the product (26 mg, $0.08 \mathrm{mmol}, 6 \%$ ) as colorless resin.

$\mathbf{R}_{\mathbf{f}}$ (pentane): 0.35 .

${ }^{1} \mathrm{H}$ NMR (400 MHz, MeOD) $\delta(\mathrm{ppm}): 5.89$ - 5.76 (m, CH2-CH-CH2, 1H), 5.45 - 5.31 (m, CH2- $\mathrm{CH}-\mathrm{CH}, 2 \mathrm{H}), 5.05-4.91$ (m, CH-CH2, 2H), 3.51 - 3.42 (m, Br-CH2-CH2, $2 \mathrm{H}), 2.15-2.01(\mathrm{~m}, \mathrm{CH} 2-\mathrm{CH} 2-\mathrm{CH}, 6 \mathrm{H}), 1.93-1.81(\mathrm{~m}, \mathrm{BrCH} 2-\mathrm{CH} 2-\mathrm{CH} 2,2 \mathrm{H}), 1.53$ $-1.32(\mathrm{~m}, \mathrm{CH} 2-\mathrm{CH} 2-\mathrm{CH} 2,16 \mathrm{H})$.

${ }^{13} \mathrm{C}$ NMR (101 MHz, MeOD) $\delta$ (ppm): 138.7 (CH2-CH-CH2), $129.8(\mathrm{CH} 2-\mathrm{CH}-\mathrm{CH})$, $129.1(\mathrm{CH} 2-\mathrm{CH}-\mathrm{CH}), 113.3(\mathrm{CH}-\mathrm{CH} 2), 33.5(\mathrm{Br}-\mathrm{CH} 2-\mathrm{CH} 2), 32.9(\mathrm{CH} 2-\mathrm{CH} 2-\mathrm{CH} 2)$, 32.5 (CH2-CH2-CH2), 29.4 (CH2-CH2-CH2), 29.2 (CH2-CH2-CH2), 28.9 (CH2-CH2- 
$\mathrm{CH} 2), 28.8(\mathrm{CH} 2-\mathrm{CH} 2-\mathrm{CH} 2), 28.7(\mathrm{CH} 2-\mathrm{CH} 2-\mathrm{CH} 2), 28.6(\mathrm{CH} 2-\mathrm{CH} 2-\mathrm{CH} 2), 27.4(\mathrm{CH} 2-$ $\mathrm{CH} 2-\mathrm{CH} 2), 26.7(\mathrm{CH} 2-\mathrm{CH} 2-\mathrm{CH} 2), 26.6(\mathrm{CH} 2-\mathrm{CH} 2-\mathrm{CH} 2) .1$ signal missing due to overlap.

GC-MS: $m / z$ calculated for [M] $\left(\mathrm{C}_{17} \mathrm{H}_{31} \mathrm{Br}\right): 314.16$, found: 314.2 .

1.2.3.2 1,1'-(Dotriaconta-6,16,26-triene-1,32-diyl)bis(3-methylimidazolium) bromide<smiles></smiles>

Grubbs Catalyst ${ }^{\circledR}$ 2nd generation (1 $\mathrm{mg}, 0.01 \mathrm{mmol}, 1 \mathrm{~mol} \%$ ) was dissolved in DCM $(1 \mathrm{~mL})$ and 17-bromoheptadeca-1,11-diene ( $39 \mathrm{mg}, 0.12 \mathrm{mmol}, 1.0$ equiv) was added. The reaction mixture was heated to $40{ }^{\circ} \mathrm{C}$ overnight. After full consumption of starting material, the reaction was concentrated in vacuo and filtered over silica. After rinsing with pentane, the solvent was concentrated and afforded a colorless resin.

This and 1-methylimidazole ( $22 \mu \mathrm{L} 0.26 \mathrm{mmol}, 2.2$ equiv) were dissolved in THF $(0.4 \mathrm{~mL})$ and stirred at $80^{\circ} \mathrm{C}$ overnight. After adding pentane, the formed white precipitate was filtered of and rinsed with pentane. The product $(27.9 \mathrm{mg}, 0.04 \mathrm{mmol}$, $33 \%$ ) was isolated as white resin.

${ }^{1} \mathrm{H}$ NMR (400 MHz, MeOD) $\delta(\mathrm{ppm}): 7.64-7.58(\mathrm{~m}, \mathrm{~N}-\mathrm{CH}-\mathrm{CH}, 2 \mathrm{H}), 7.58-7.53(\mathrm{~m}, \mathrm{~N}-$ $\mathrm{CH}-\mathrm{CH}, 2 \mathrm{H}), 5.46-5.29(\mathrm{~m}, \mathrm{CH} 2-\mathrm{CH}-\mathrm{CH}, 6 \mathrm{H}), 4.23-4.14(\mathrm{~m}, \mathrm{~N}-\mathrm{CH} 2-\mathrm{CH} 2,4 \mathrm{H}), 3.91$ (s, CH3-N, 6H), $2.06-1.93(\mathrm{~m}, \mathrm{CH} 2-\mathrm{CH} 2-\mathrm{CH}, 12 \mathrm{H}), 1.92-1.83(\mathrm{~m}, \mathrm{NCH} 2-\mathrm{CH} 2-\mathrm{CH} 2$, $4 \mathrm{H}), 1.47-1.22(\mathrm{~m}, \mathrm{CH} 2-\mathrm{CH} 2-\mathrm{CH} 2,34 \mathrm{H})$.

${ }^{13} \mathrm{C}$ NMR (101 MHz, MeOD) $\delta$ (ppm): $134.6(\mathrm{CH} 2-\mathrm{CH}-\mathrm{CH}), 134.2(\mathrm{CH} 2-\mathrm{CH}-\mathrm{CH}), 133.5$ (CH2-CH-CH), $127.5(\mathrm{~N}-\mathrm{CH}-\mathrm{CH}), 126.2(\mathrm{~N}-\mathrm{CH}-\mathrm{CH}), 53.4(\mathrm{~N}-\mathrm{CH} 2-\mathrm{CH} 2), 39.0(\mathrm{CH} 3-$ $\mathrm{N}), 36.2(\mathrm{CH} 2), 35.9(\mathrm{CH} 2), 33.6(\mathrm{CH} 2), 33.2(\mathrm{CH} 2), 32.7(\mathrm{CH} 2), 29.3(\mathrm{CH} 2) .6$ signals missing due to overlap.

HRMS (ESI): $\mathrm{m} / \mathrm{z}$ calculated for $[\mathrm{M}]^{2+}\left(\mathrm{C}_{40} \mathrm{H}_{70} \mathrm{~N}_{4}{ }^{2+}\right): 303.2800$, found: 303.2791 . 


\subsection{Experimental Spectra}

\subsubsection{NMR Spectra}
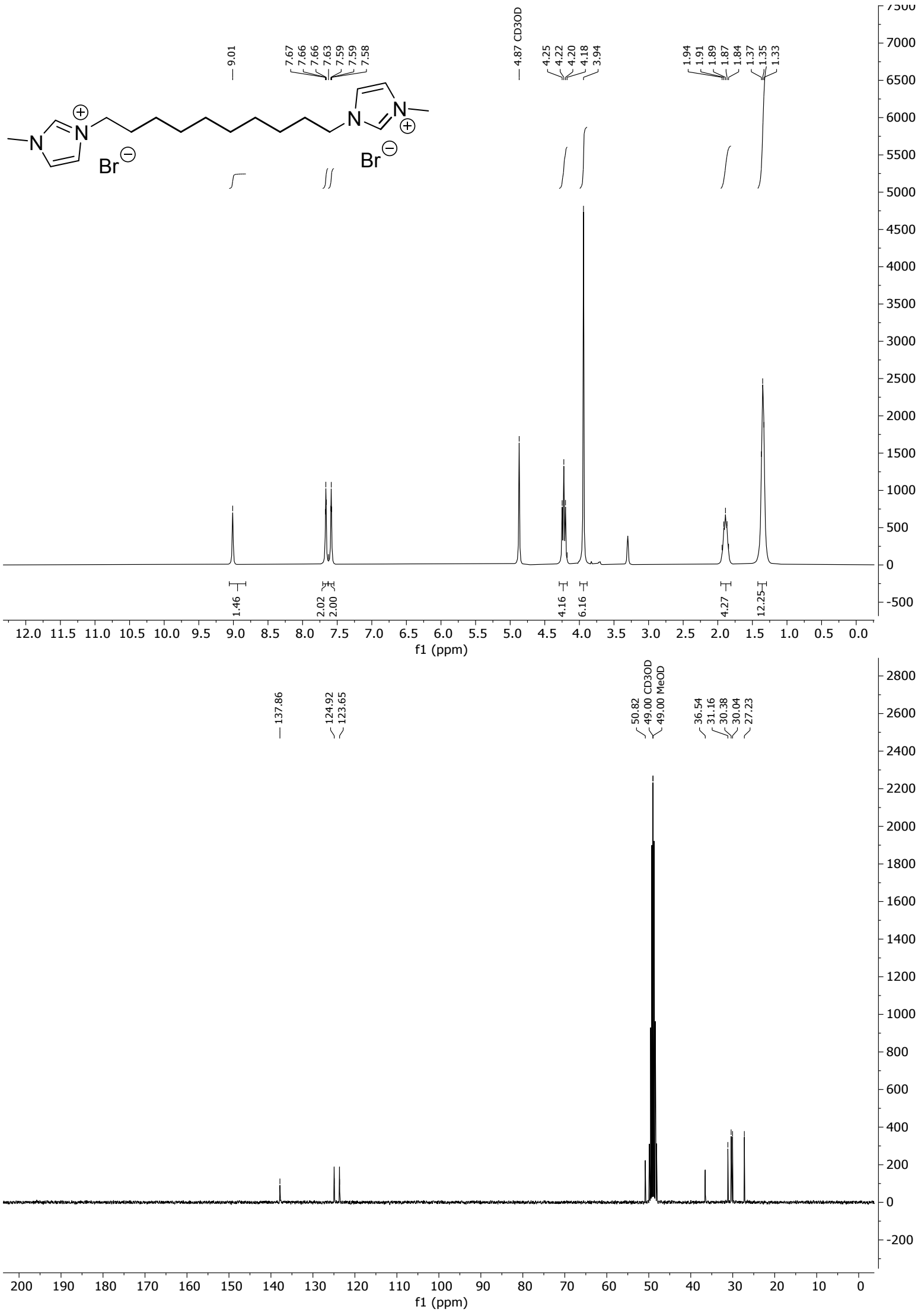

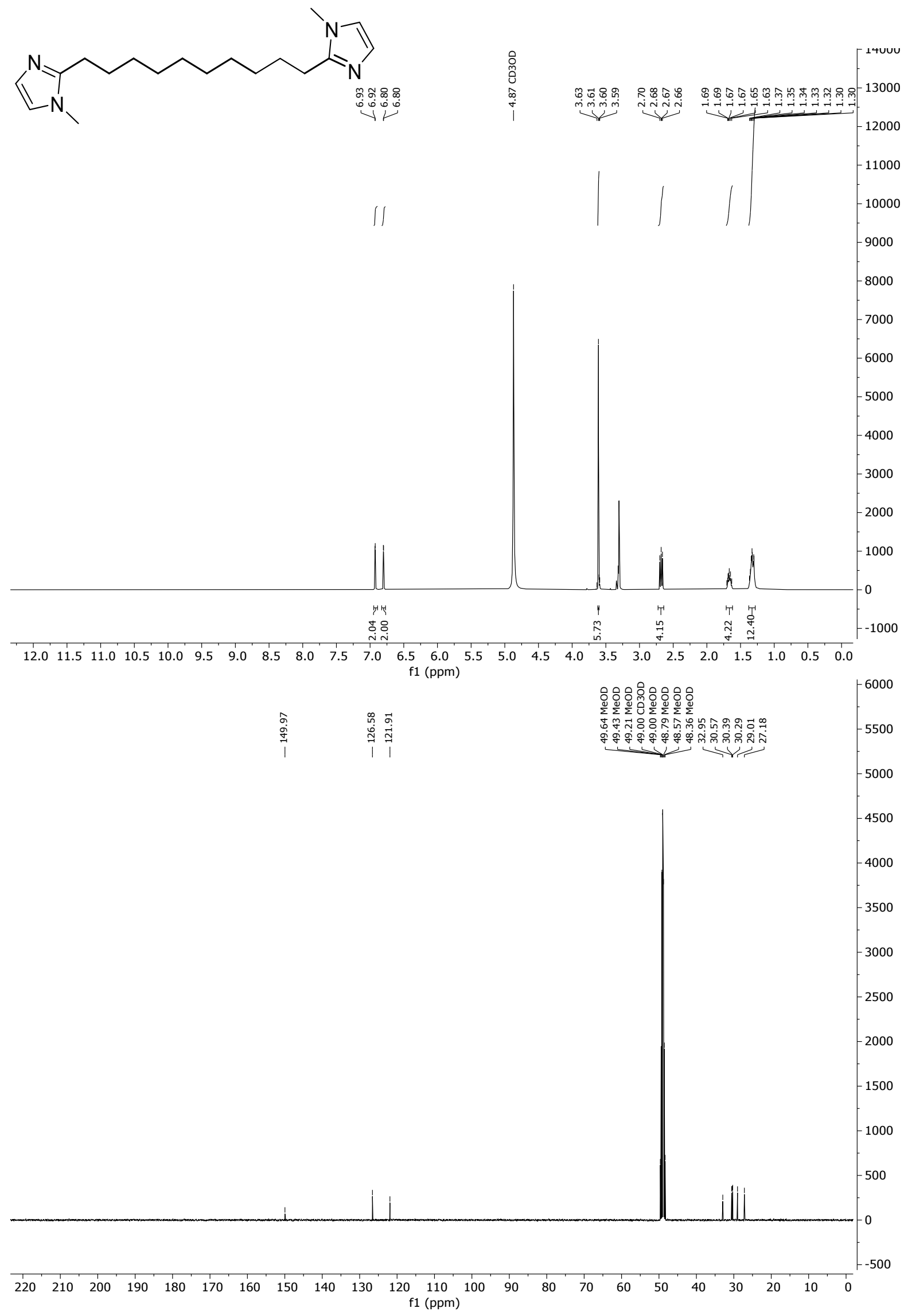

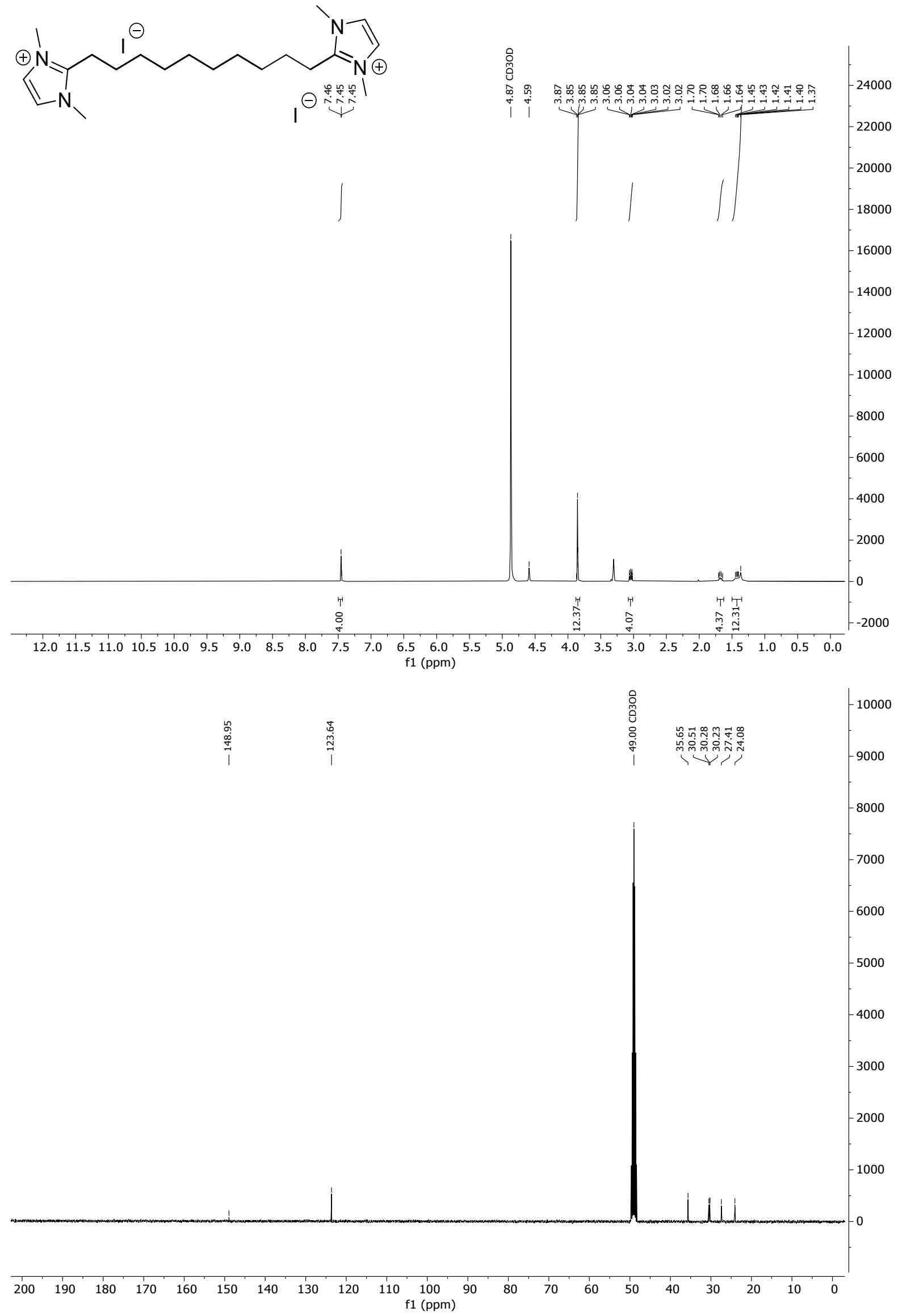

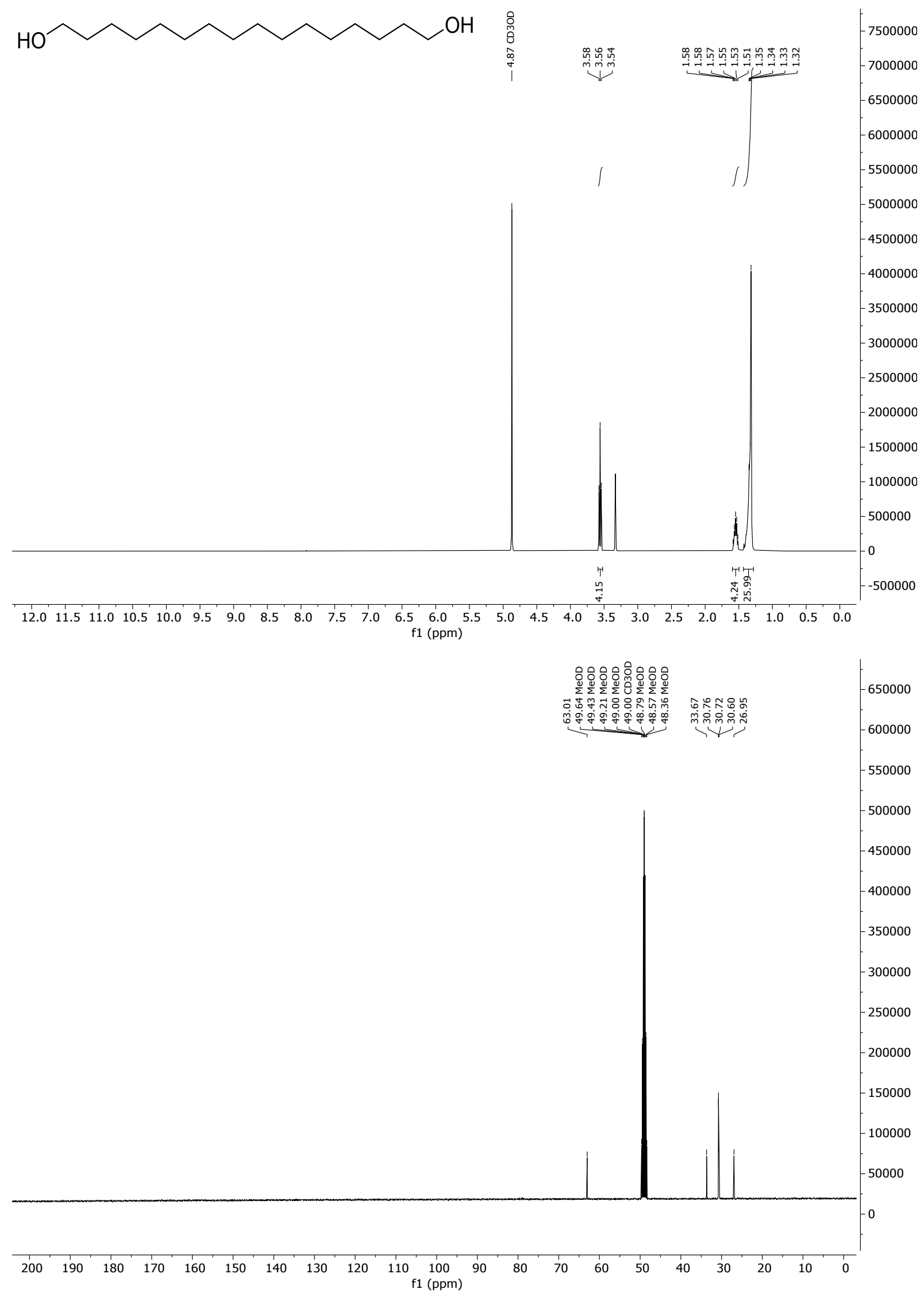


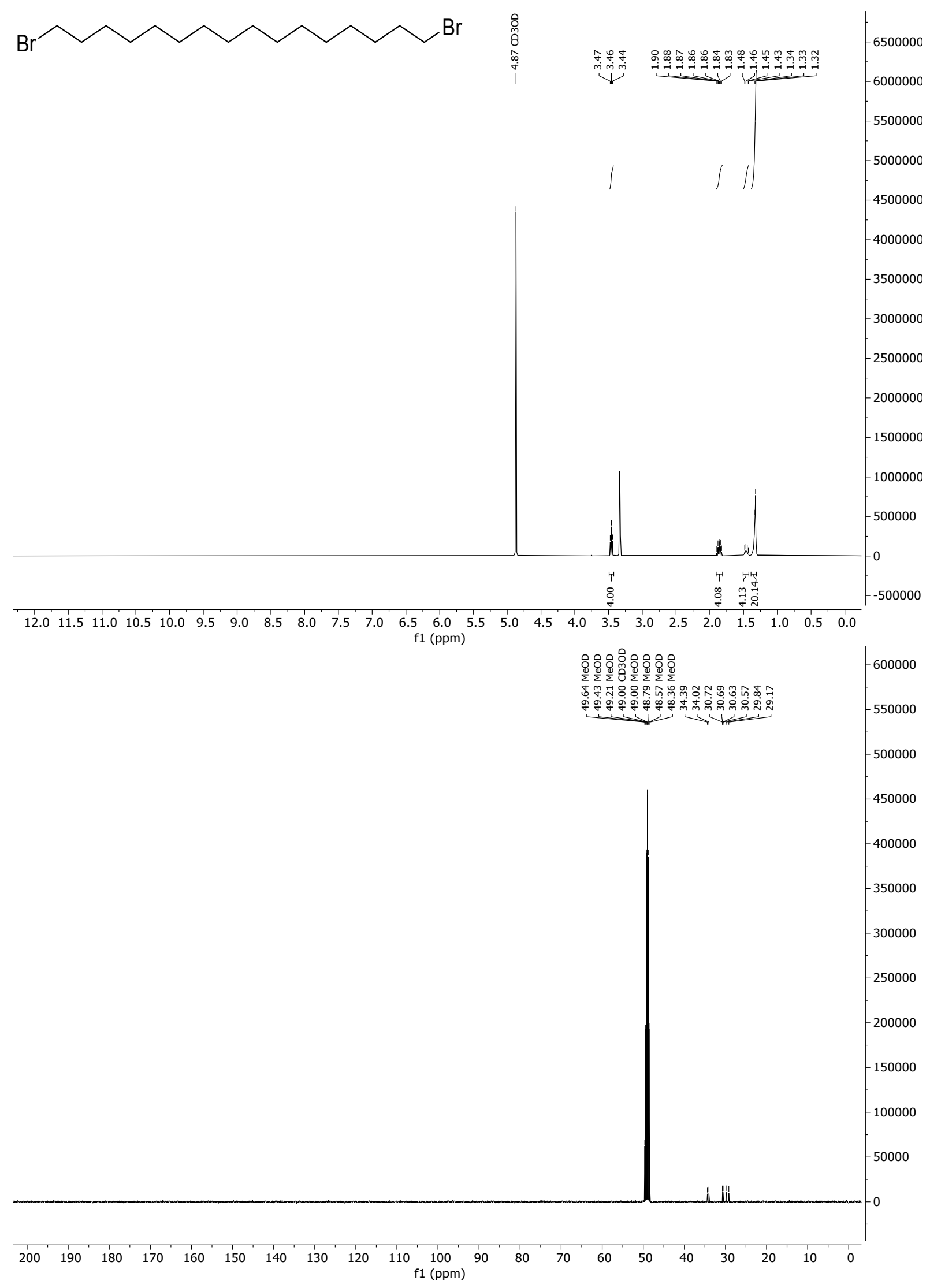




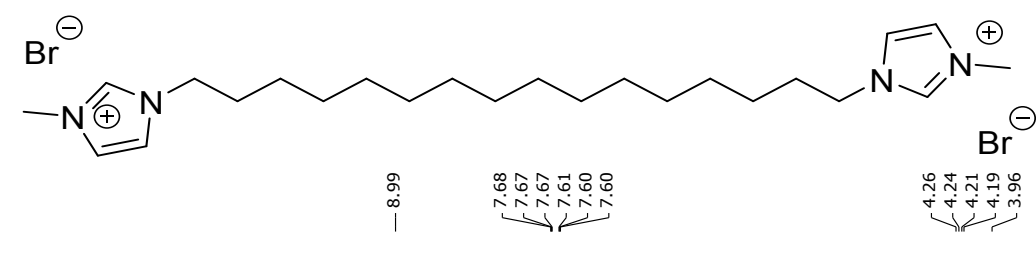

$$
\sqrt{\mathrm{Br}^{\ominus}}
$$

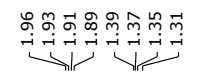

$-8500$

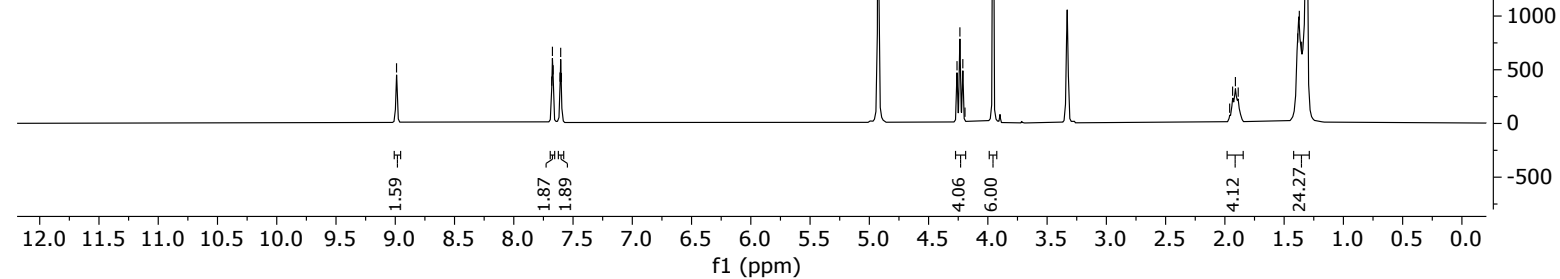

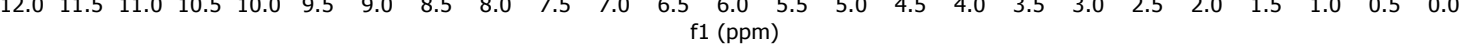

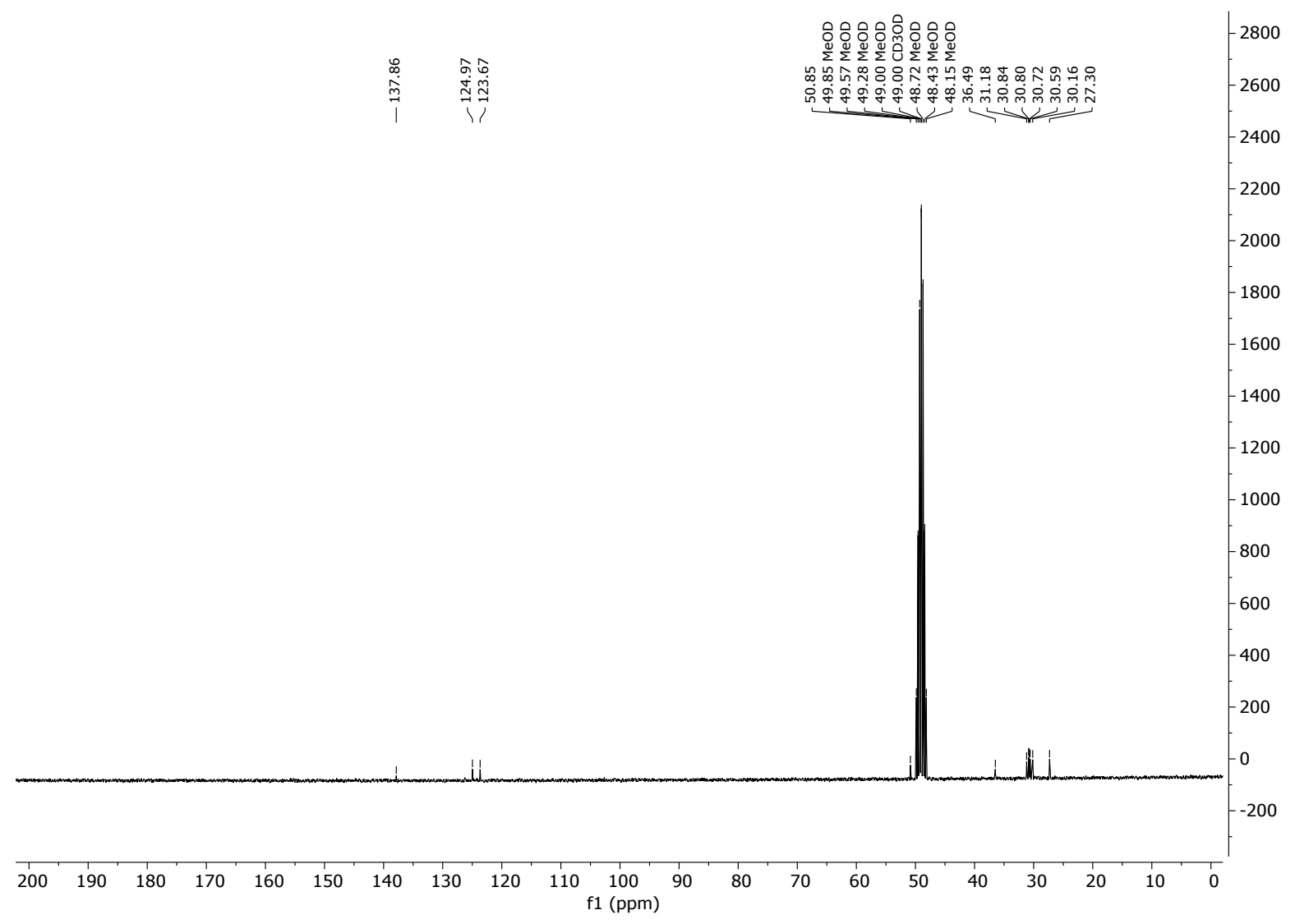



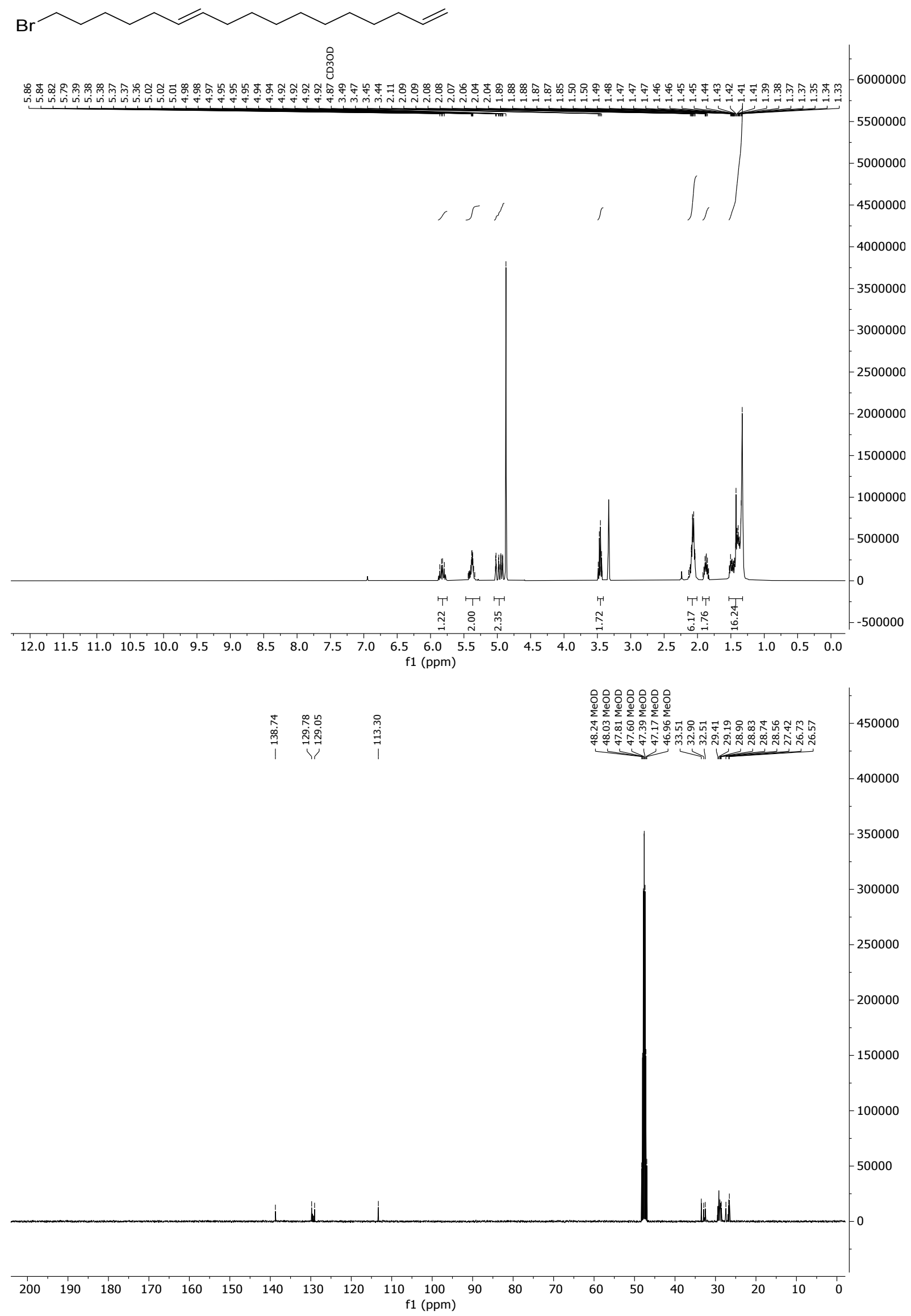

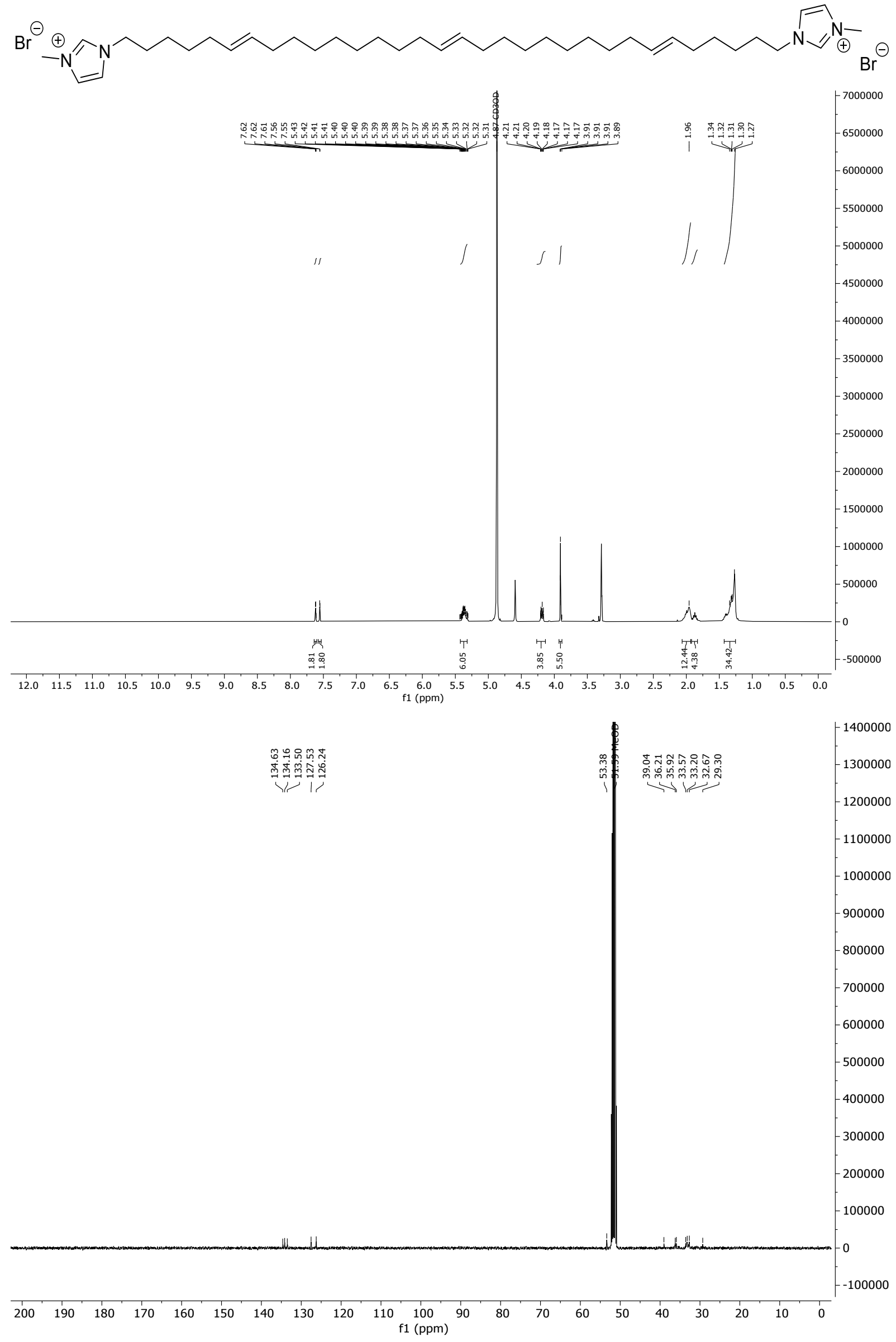


\subsubsection{UPLC-MS Data}
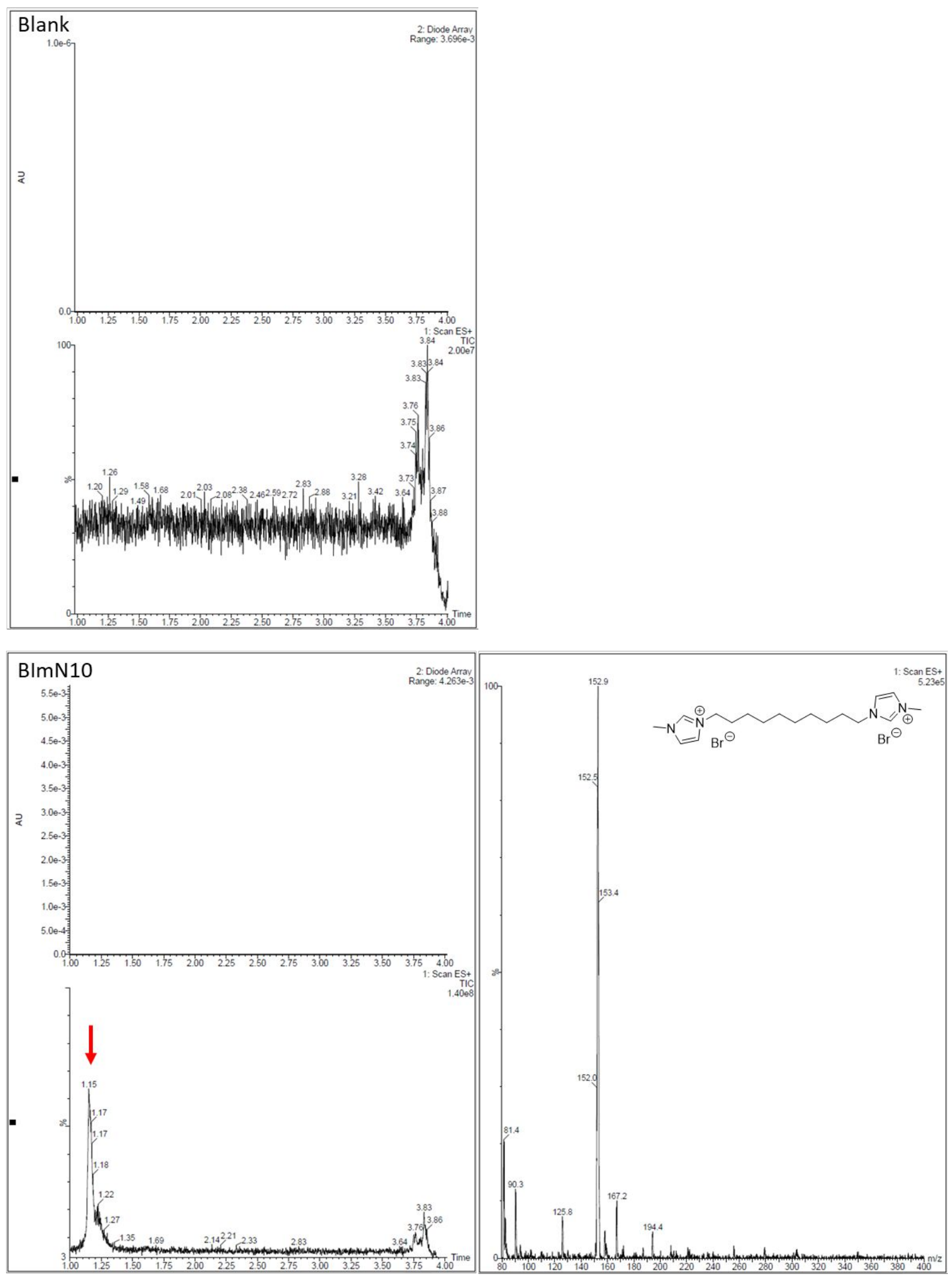

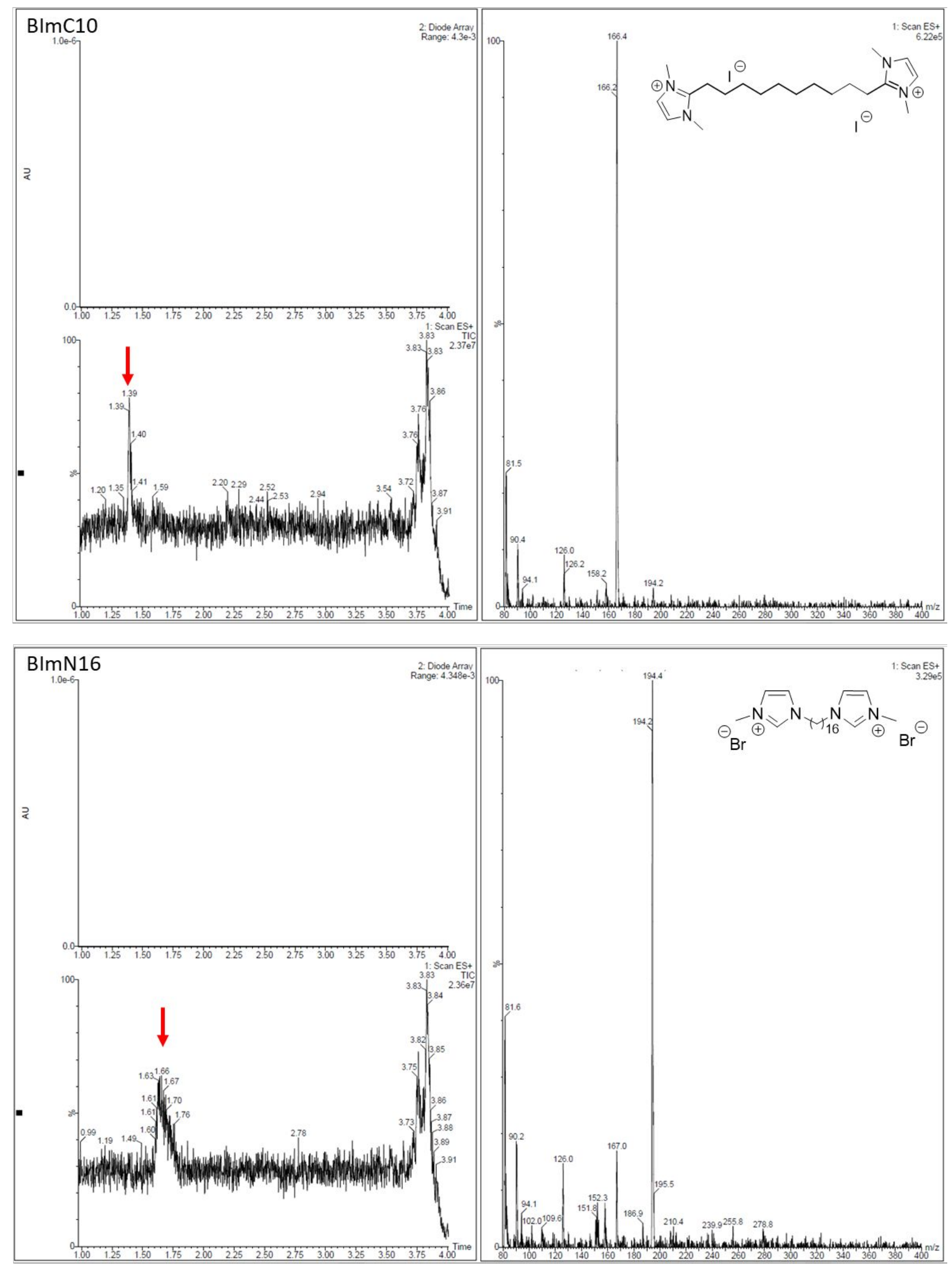


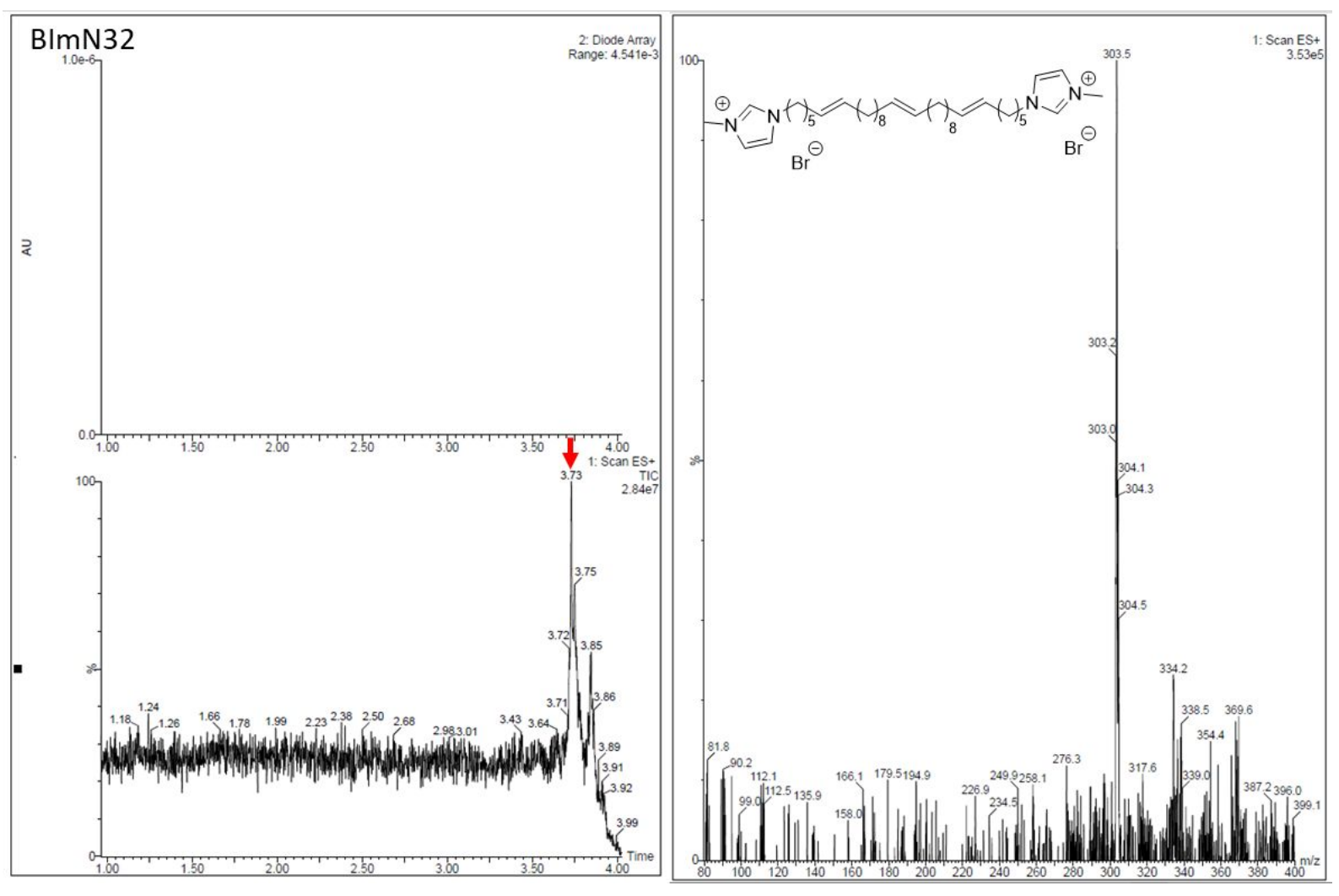




\section{Additional Figures}

a)

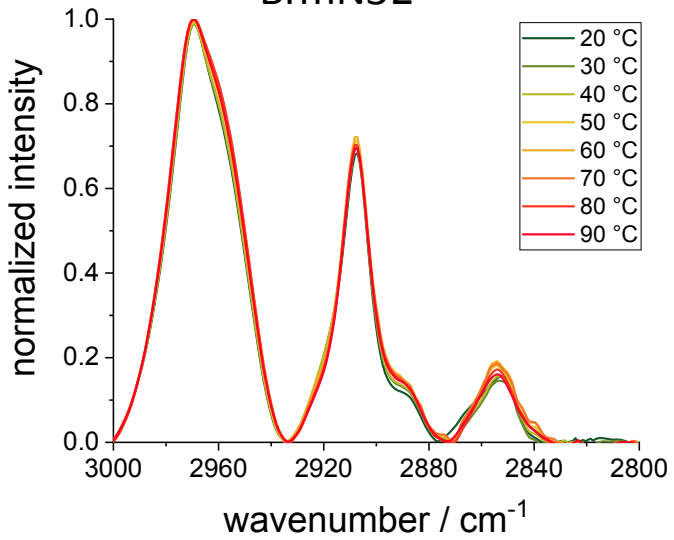

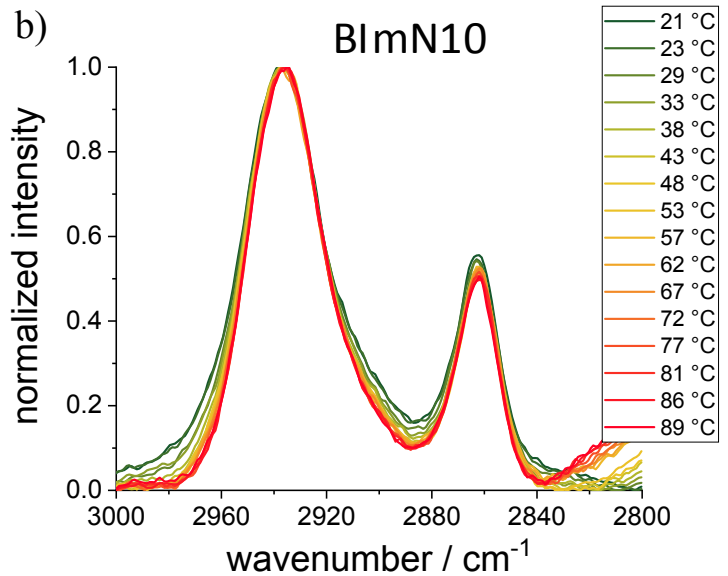

Figure SI 1. Wavenumber, $\tilde{\boldsymbol{v}}$, of the symmetric and asymmetric $\mathrm{CH}_{2}$ vibrational band of a) BImN32 and b) BImN10.
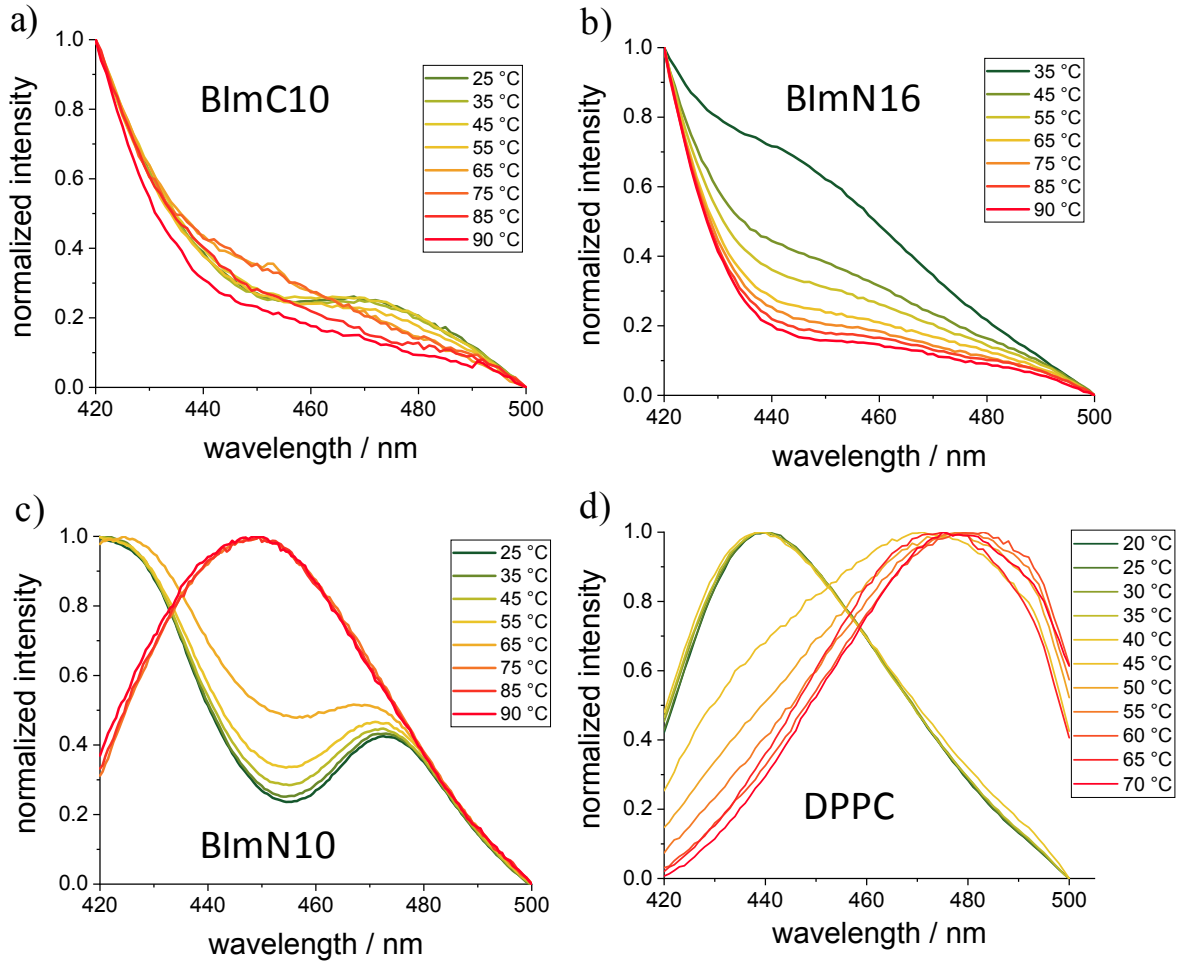

Figure SI 2. Laurdan fluorescence spectra as a function of temperature of a) BImC10, b) BImN16, c) BImN10, and d) DPPC. 
a)

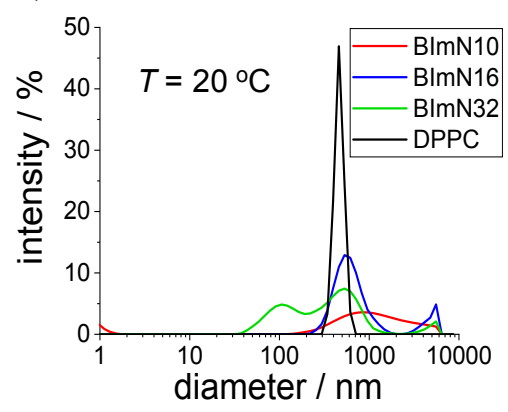

c)

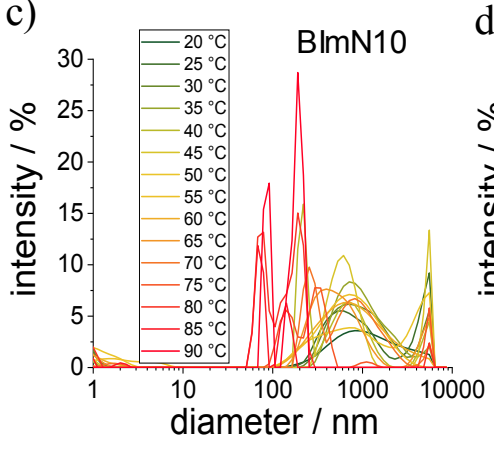

b)

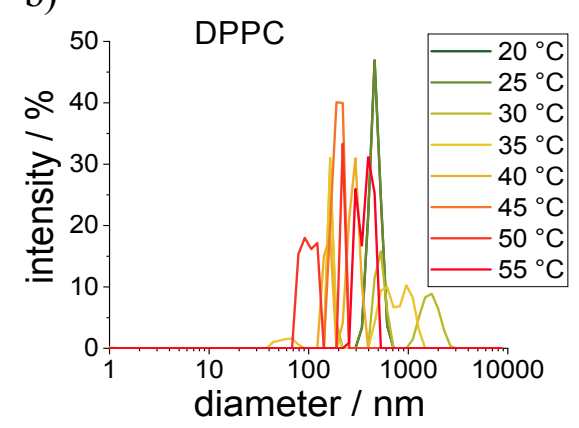

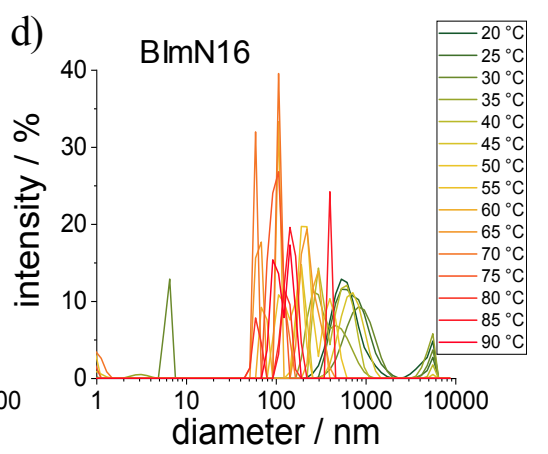

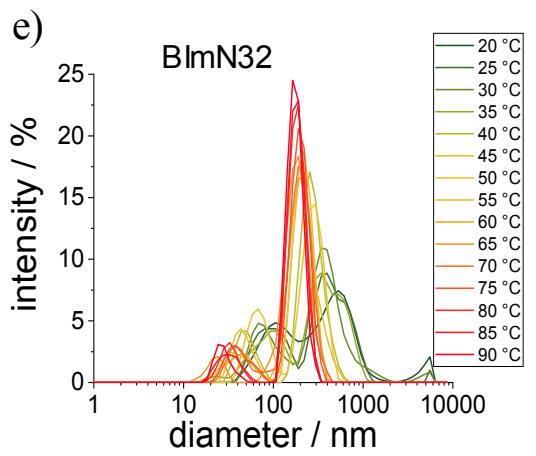

Figure SI 3. DLS experiments: Diameter distribution of the different nitrogen linked BIm salts and DPPC, a) at $20{ }^{\circ} \mathrm{C}$ for comparison, b) for DPPC, c) for BImN10, d) for BImN16, and e) for BImN32 as a function of temperature.

a)

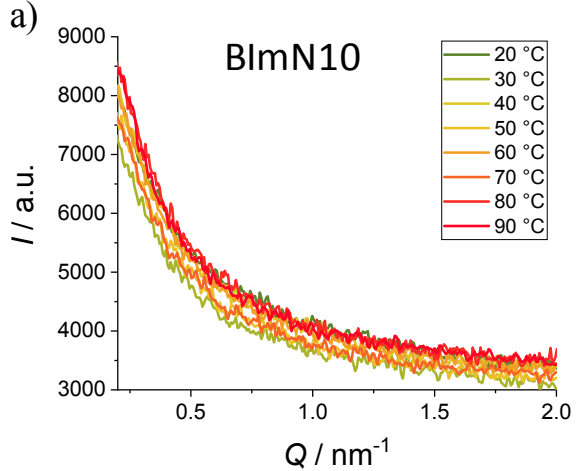

c)

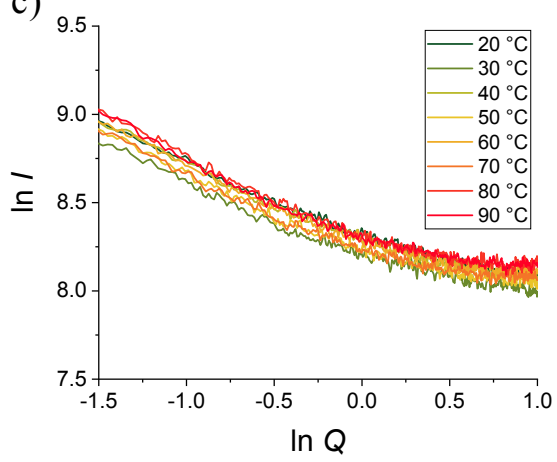

b)

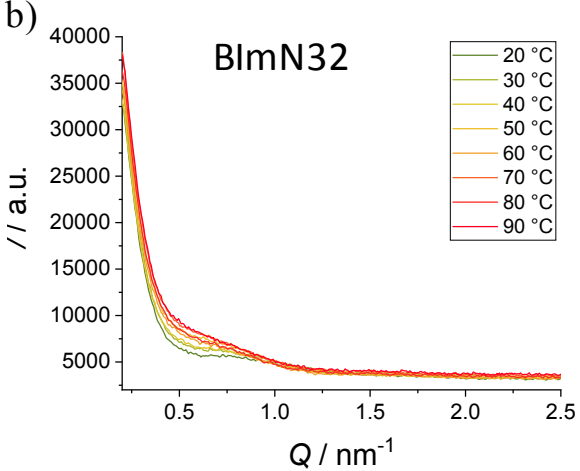

d)

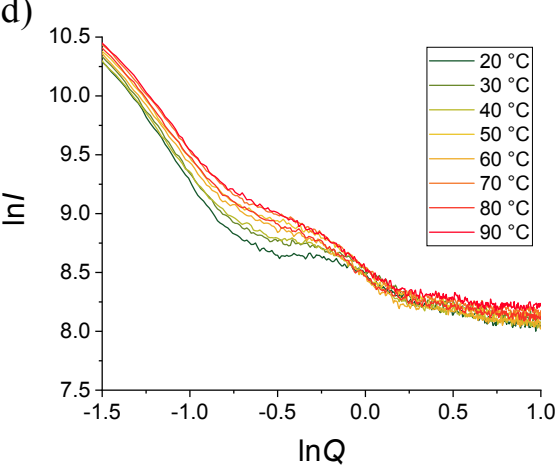

Figure SI 4. SAXS patterns $I(Q)$ and their double logarithmic plots of a) BImN10 and b) BImN32 as a function of temperature. 
brightfield
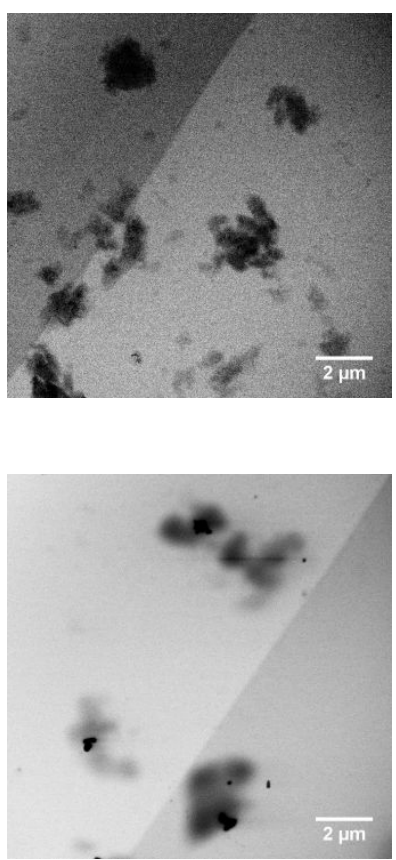

darkfield
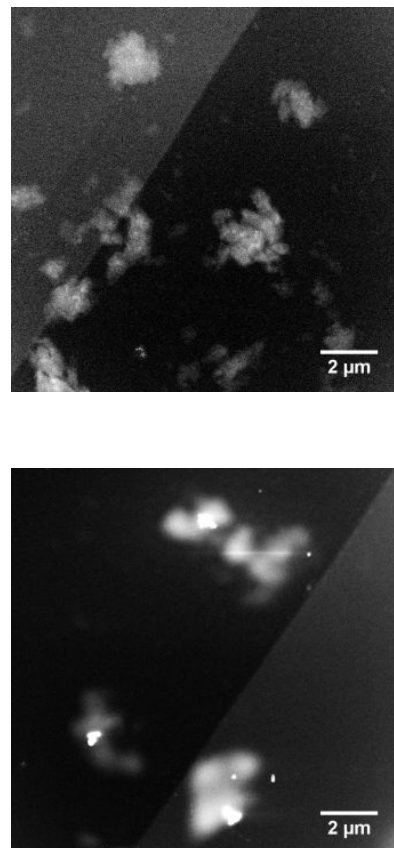

Figure SI 5. In situ liquid-cell TEM pictures of BImN32 in brightfield (left) und the darkfield (right). The scale bar represents $2 \mu \mathrm{m}$.

a)

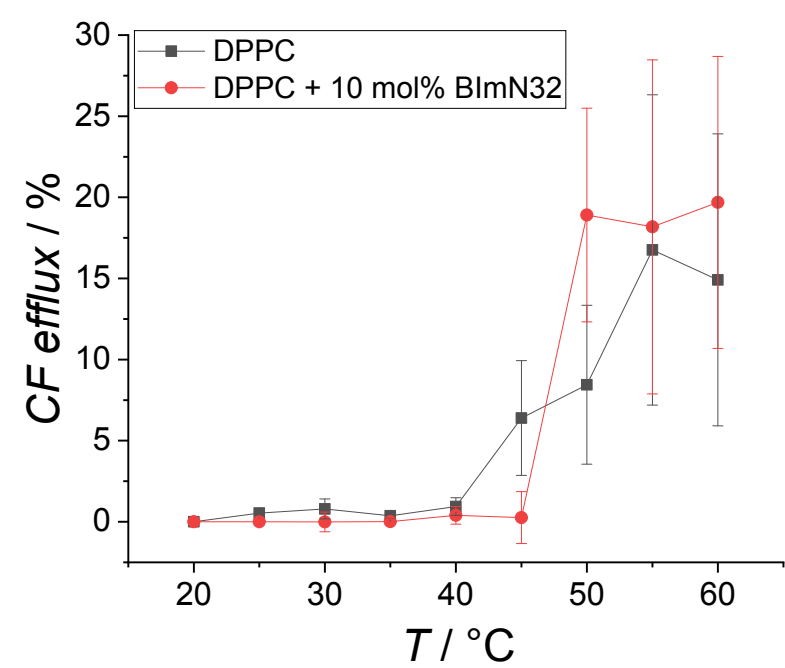

b)

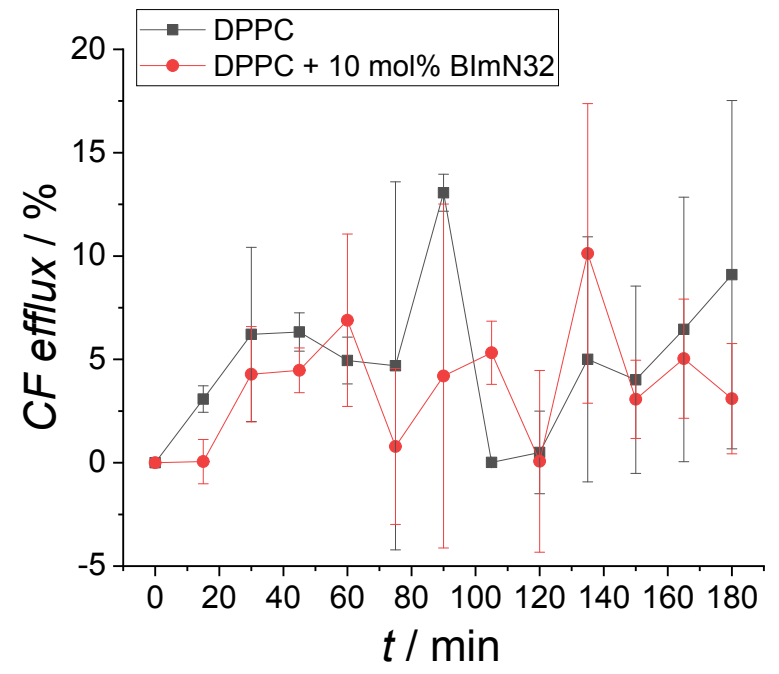

Figure SI 6. Leakage test using lipid bilayers consisting of $5 \mathrm{mg} / \mathrm{mL}$ DPPC and DPPC + 10 mol\% BImN32 measured by carboxyfluorescein (CF) efflux a) below and above the gel-to-fluid phase transition (heating scan), b) time dependent at $T=25^{\circ} \mathrm{C}$. The incorporation of BImN32 into the DPPC bilayer does not lead to an increased efflux of the fluorophore. 
a)
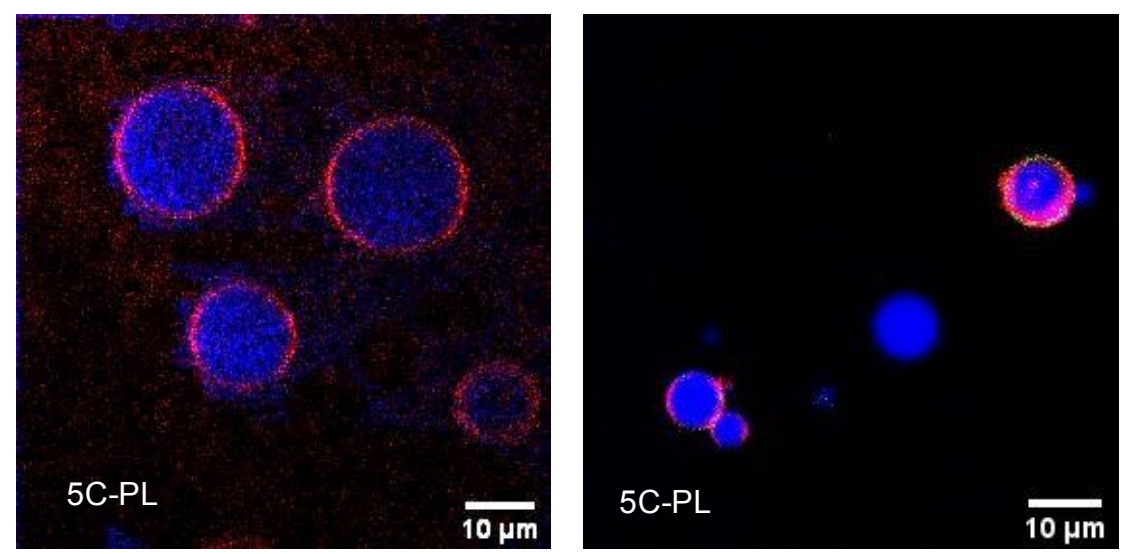

b)
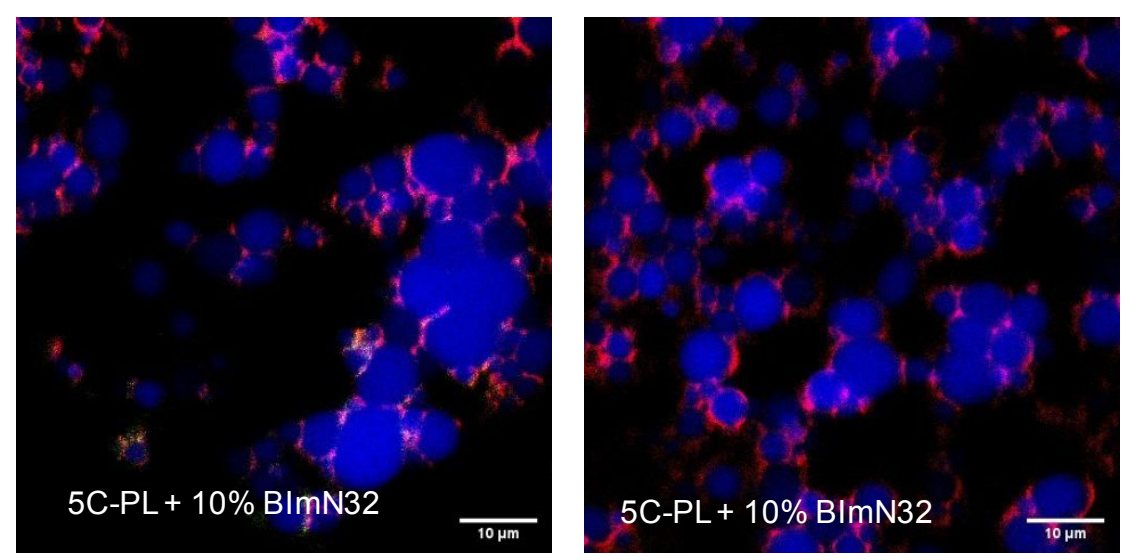

Red: N-Rhodamin DHPE Blue: Atto647

Figure SI 7. Selected confocal microscopy cross-sectional images of the 5C-PL phospholipid bilayer system DPPC:DPPG:DOPC:DOPG:cholesterol (45:5:20:5:25 $\mathrm{mol} \%, 1 \mathrm{mM}$ in total) used for the leakage test. The red fluorescence originates from the fluorescence marker $N$-Rh-DHPE ( $I_{d}$-Phase marker) while the blue fluorescence is due to Atto647 incorporated into the interior of the GUVs and washed from the buffer after GUV formation ( $T=25^{\circ} \mathrm{C}$ ). The incorporation of BImN32 into the PL system does not lead to leakage of the fluorophore.

\section{References}

[1] Loscher, S.; Schobert, R. Total Synthesis and Absolute Configuration of Epicoccamide D, a Naturally Occurring Mannosylated 3-Acyltetramic Acid. Chem. Eur. J. 2013, 19, 10619-10624.

[2] Braun, M.; Camps, X.; Vostrowsky, O.; Hirsch, A.; Endreß, E.; Bayerl, T. M.; Birkert, O.; Gauglitz, G. Synthesis of a Biotinated Lipofullerene as a New Type of Transmembrane Anchor. Eur. J. Org. Chem. 2000, 7, 1173-1181.

[3] Castilla, J.; Rísquez, R.; Higaki, K.; Nanba, E.; Ohno, K.; Suzuki, Y.; Díaz, Y.; Mellet, O. C.; García Fernández, J. M. G.; Castillón, S. Conformationally-locked $\mathrm{N}$-glycosides: Exploiting long-range non-glycone interactions in the design of 
pharmacological chaperones for Gaucher disease. Eur. J. Med. Chem. 2015, 90 258-266.

[4] Lafleur, R. P. M.; Herziger, S.; Schoenmakers, S. M. C.; Keizer, A. D. A.; Jahzerah, J.; Thota, B. N. S.; Su, L.; Bomans, P. H. H.; Sommerdijk, N. A. J. M.; Palmans, A. R. A.; Haag, R.; Friedrich, H.; Böttcher, C.; Meijer, E. W. J. Am. Chem. Soc. 2020, 142, 17644-17652. 